\title{
Maximum Norm Estimates in the Finite Element Method on Plane Polygonal Domains. Part 1
}

\author{
By A. H. Schatz and L. B. Wahlbin*
}

\begin{abstract}
The finite element method is considered when applied to a model Dirichlet problem on a plane polygonal domain. Rate of convergence estimates in the maximum norm, up to the boundary, are given locally. The rate of convergence may vary from point to point and is shown to depend on the local smoothness of the solution and on a possible pollution effect. In one of the applications given, a method is proposed for calculating the first few coefficients (stress intensity factors) in an expansion of the solution in singular functions at a corner from the finite element solution. In a second application the location of the maximum error is determined.
\end{abstract}

A rather general class of non-quasi-uniform meshes is allowed in our present investigations. In a subsequent paper, Part 2 of this work, we shall consider meshes that are refined in a systematic fashion near a corner and derive sharper results for that case.

0. Introduction. Let $\Omega$ be a bounded simply connected domain in the plane with boundary $\partial \Omega$ consisting of a finite number of straight line segments meeting at vertices $v_{j}, j=1, \ldots, M$, of interior angles $0<\alpha_{1} \leqslant \cdots \leqslant \alpha_{M}<2 \pi$ (in a suitable ordering). We shall consider the Dirichlet problem

$$
\begin{cases}-\Delta u=f & \text { in } \Omega, \\ u=0 & \text { on } \partial \Omega,\end{cases}
$$

where $f$ is a given function, which for simplicity we assume to be smooth.

To solve the problem $(0.1)$ numerically, let $S^{h}=S^{h}(\Omega), 0<h<1$, denote a one-parameter family of finite dimensional subspaces of $\stackrel{\circ}{H}^{1}(\Omega) \cap W_{\infty}^{1}(\Omega)$. We have in mind piecewise polynomials of a fixed degree on a sequence of partitions of $\Omega$. In our considerations the partitions do not have to be quasi-uniform, not even locally (cf. examples in Section 9).

Let $u_{h} \in S^{h}$ be the approximate solution of $(0.1)$ defined by the relation

$$
A\left(u_{h}, \chi\right)=(f, \chi) \text { for all } \chi \in S^{h} .
$$

Here $A(v, w)=\int_{\Omega} \nabla v \cdot \nabla w d x$, and $(v, w)=\int_{\Omega} v w d x$.

We wish to obtain local estimates up to the boundary in the maximum norm for the error $u-u_{h}$. Although our present assumptions allow meshes that are refined near a corner, in the subsequent paper, Part 2, we shall investigate the error in more detail in that case, and obtain sharper results. The general results derived in the present paper will be essential in those investigations. 
Since the ability of a given subspace to approximate a given function depends in part on the regularity of the function, let us first discuss the regularity of the solution of (0.1). It is well known that even if $f$ is smooth, $u$ may be "badly" behaved near the corners. In fact, if we introduce polar coordinates $(\rho, \theta)$ at the vertex $v_{j}$ so that the interior of the wedge is given by $0<\theta<\alpha_{j}$ and set $\beta_{j}=\pi / \alpha_{j}$, then near $v_{j}$ the solution $u$ behaves like

$$
u(\rho, \theta)=k_{j} \rho^{\beta_{j}}\left(\ln \frac{1}{\rho}\right)^{m}{ }^{j} \sin \left(\beta_{j} \theta\right)+\text { smoother terms. }
$$

Here $k_{j}$ is a constant, and $m_{j}=0$ unless $\beta_{j}=2,3, \ldots$. Globally one can then say that for any $\epsilon>0, u \in H^{1+\beta_{M^{-}} \epsilon}(\Omega)$ or $C^{\beta_{M}-\epsilon}(\bar{\Omega})$. If we let $\Omega_{j}, j=1, \ldots, M$, denote the intersection of $\Omega$ with a disc centered at the $j$ th vertex and such that $\Omega_{j}$ contains no other vertex, and set $\Omega_{0}=\Omega \backslash\left(\bigcup_{j=1}^{M} \bar{\Omega}_{j}\right)$ then we have $u \in H^{1+\beta_{j}-\epsilon}\left(\Omega_{j}\right)$ or $C^{\beta_{j}-\epsilon}\left(\bar{\Omega}_{j}\right), j=1, \ldots, M$, and $u \in C^{\infty}\left(\Omega_{0}\right)$.

For many finite element spaces the following holds: Let $r \geqslant 2$ be an integer (one may, e.g. take piecewise polynomials of degree $r-1$ ). Then there exists a constant $C$ and a $\chi \in S^{h}(\Omega)$ such that

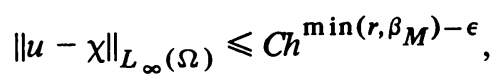

and

$$
\left.\|u-\chi\|_{H^{1}(\Omega)} \leqslant C h^{\min (r-1, \beta}\right)^{-\epsilon} .
$$

In fact, $\chi$ has the additional local properties that

$$
\begin{gathered}
\|u-\chi\|_{L_{\infty}\left(\Omega_{j}\right)} \leqslant C h^{\min \left(r, \beta_{j}\right)-\epsilon}, \\
\|u-\chi\|_{H^{1}\left(\Omega_{j}\right)} \leqslant C h^{\min \left(r-1, \beta_{j}\right)-\epsilon}, \quad j=1,2, \ldots, M,
\end{gathered}
$$

and since $u \in C^{\infty}\left(\Omega_{0}\right)$,

$$
\|u-\chi\|_{L_{\infty}\left(\Omega_{0}\right)} \leqslant C h^{r}
$$

Let us remark that if $\beta_{j} \leqslant r-1$, as is always the case when $1 / 2<\beta_{j}<1$, i.e., $\alpha_{j}$ is concave, then the estimates $(0.6)$ and $(0.7)$ essentially yield the same degree of approximation in $L_{\infty}\left(\Omega_{j}\right)$ and $H^{1}\left(\Omega_{j}\right)$, respectively.

Before discussing our results, let us describe some other recent work on maximum norm estimates which are relevant to our work here. For other references concerning the finite element method on domains with corners, we refer, e.g. to Babuška [1], Babuška and Aziz [2], Babuška and Rheinboldt [4], Babuška and Rosenzweig [5], Eisenstat and Schultz [11], and Thatcher [36].

Maximum norm estimates for the finite element method on irregular but quasiuniform meshes have been discussed in Douglas, Dupont and Wheeler [10], Frehse and Rannacher [12], Natterer [21], Nitsche [24], [25], Schatz and Wahlbin [31], and Scott [32]. (In these papers, references to work concerning maximum norm estimates on uniform meshes can be found.) In particular the papers [21], [12] and [24] give global estimates for the error on a convex polygonal domain (i.e., $\beta_{M}>1$ ) where the 
subspaces $S^{h}$ are piecewise linear functions defined on a quasi-uniform triangulation of $\Omega$. Improving the estimates in [21] and [12], Nitsche shows that for any $\chi \in S^{h}$,

$$
\left\|u-u_{h}\right\|_{L_{\infty}(\Omega)} \leqslant h \ln \frac{1}{h}\|u-\chi\|_{W_{\infty}^{1}(\Omega)} .
$$

Since in this case $\Omega$ is convex, we may deduce from the counterpart of $(0.4)$ in $W_{\infty}^{1}$ the global estimate

$$
\left\|u-u_{h}\right\|_{L_{\infty}(\Omega)} \leqslant C h^{\min \left(r, \beta_{M}\right)-\epsilon} .
$$

One of the shortcomings of a global analysis of problems where the regularity of the solution may vary in the domain is that it will in general yield a rate of convergence commensurate with the worst behavior of the solution. Sharper results can often be obtained by a local analysis. For example, if $\Omega$ is a polygonal domain (not necessarily convex) and $S^{h}$ is taken to be any one of a rather general class of finite elements defined on a quasi-uniform partition of $\Omega$, then as a special case of the results of [31] we have that for interior subdomains $\Omega_{0} \subset \subset \Omega_{0}^{\prime} \subset \subset \Omega$ and any $\chi \in S^{h}$,

$$
\left\|u-u_{h}\right\|_{L_{\infty}\left(\Omega_{0}\right)} \leqslant C\left[\left(\ln \frac{1}{h}\right)^{\bar{r}}\|u-\chi\|_{L_{\infty}\left(\Omega_{0}^{\prime}\right)}+\left\|u-u_{h}\right\|_{-p, \Omega_{0}^{\prime}}\right] .
$$

Here $\bar{r}=1$ if $r=2$ and $\bar{r}=0$ if $r \geqslant 3$. The number $p$ is an arbitrary positive integer and $\|\cdot \mid\|_{-p, \Omega_{0}^{\prime}}$ denotes the norm dual to $\stackrel{\circ}{H}^{p}\left(\Omega_{0}^{\prime}\right)$. The error is thus divided into two parts. The first depends on the local smoothness properties of $u$ and the ability of the subspace to approximate locally. From (0.8) we have

$$
\|u-\chi\|_{L_{\infty}\left(\Omega_{0}^{\prime}\right)} \leqslant C h^{r} \text {. }
$$

The second term in (0.10) is the error in an arbitrary negative norm Sobolev space. This term measures the effect on the local error of such things as the smoothness of the solution outside of $\Omega_{0}^{\prime}$, the smoothness of the boundary, etc. and may be estimated by using a duality argument. In Lemma 4.2 we shall show that for $p>\beta_{M}-1$,

$$
\left\|u-u_{h}\right\|_{-p, \Omega} \leqslant C h^{\min \left(2(r-1), 2 \beta_{M}\right)-\epsilon} .
$$

Since $2(r-1) \geqslant r$ we have from $(0.10),(0.11)$ and $(0.12)$ that

$$
\left\|u-u_{h}\right\|_{L_{\infty}\left(\Omega_{0}\right)} \leqslant C h^{\min \left(r, 2 \beta_{M}\right)-\epsilon} .
$$

We first notice that in many cases the estimate $(0.13)$ is "better" than $(0.9)$, even in the convex case. Next we see that in comparison to $(0.8)$ the estimate $(0.13)$ may not be optimal. For example, if $1 / 2<\beta_{M}<1$ (i.e., a concave maximal angle) then $2 \beta_{M}<r$ and therefore $\left\|u-u_{h}\right\|_{L_{\infty}\left(\Omega_{0}\right)} \leqslant C h^{2 \beta_{M}-\epsilon}$. This is the well-known pollution effect due to the corner of maximal angle-it has been observed in calculations.

As remarked before, the aim of this paper is to provide local estimates up to the boundary for a polygonal domain (not necessarily convex) using a general class of subspaces. We wish to emphasize here that once having such estimates we will then be able to consider other questions which are of interest, namely: 1. the calculation of the coefficients (stress intensity factors) in the expansion (0.3), and 2. the location of the maximum error in $\Omega$. 
Let us now briefly describe our results. We shall refer the reader to the appropriate place in the text for more precise statements.

In Theorem 4.1 we shall show that with $\Omega_{j}, j=1, \ldots, M$, and $\Omega_{0}$ as above,

$$
\left\|u-u_{h}\right\|_{L_{\infty}\left(\Omega_{j}\right)} \leqslant C h^{\min \left(r, \beta_{j}, 2 \beta_{M}\right)-\epsilon}, \quad j=1, \ldots, M .
$$

and

$$
\left\|u-u_{h}\right\|_{L_{\infty}\left(\Omega_{0}\right)} \leqslant C h^{\min \left(r, 2 \beta_{M}\right)-\epsilon} .
$$

Except for the term $2 \beta_{M}$ occurring in the exponent, which corresponds to a possible pollution effect due to the corner of maximal interior angle, these estimates are analogous to $(0.6),(0.8)$.

The results (0.14) and (0.15), and others to be given below, are derived as consequences of the following two basic estimates, $(0.16)$ and $(0.17)$ below. Let $D_{1} \subseteq$ $D \subseteq \Omega$ be such that roughly speaking the distance $d$ from $D_{1}$ to the part of the boundary of $D$ which $D$ does not have in common with $\Omega$ is greater or equal to $C h^{1-\delta}$ for given $\delta>0$. If the mesh is quasi-uniform, $\delta$ may be taken equal zero. In Theorem 3.2 we show that if $D$ does not contain any concave corners, then for any $\chi \in S^{h}$,

$$
\begin{aligned}
& \left\|u-u_{h}\right\|_{L_{\infty}\left(D_{1}\right)} \\
& \quad \leqslant C h^{-\epsilon}\left(\|u-\chi\|_{L_{\infty}(D)}+h\|\nabla(u-\chi)\|_{L_{\infty}(D)}+d^{-1-p}\left\|u-u_{h}\right\|_{-p, D}\right) .
\end{aligned}
$$

More generally if $D_{1} \subseteq D \subseteq \Omega$ are two domains with $d \geqslant C h^{1-\delta}$ (and $D$ may contain a concave corner), then for any $\chi \in S^{h}$ we have, Theorem 3.1,

$$
\begin{aligned}
\left\|u-u_{h}\right\|_{L_{\infty}\left(D_{1}\right)} \leqslant C h^{-\epsilon}\left(\|u-\chi\|_{L_{\infty}(D)}+d^{-1}\|u-\chi\|_{L_{2}(D)}\right. \\
\left.+\|\nabla(u-\chi)\|_{L_{2}(D)}+d^{-1-p}\left\|u-u_{h}\right\|_{-p, D}\right) .
\end{aligned}
$$

Remark 0.1 . The results $(0.16)$ and (0.17) are valid for $u-u_{h}$ which satisfy (0.1) and $(0.2)$ locally on a domain $D$. Thus these results may be used to obtain local estimates for the error, when for example: 1 . Neumann or mixed boundary conditions are imposed on the part of the boundary outside of $D$. 2. the boundary outside of $D$ is not necessarily polygonal.

Let us further point out that the proof of $(0.16)$ essentially consists of extending the techniques of [31]. The proof of (0.17) follows by converting a local $H^{1}$ estimate, cf. [27], to an $L_{\infty}$ estimate by means of an inverse relation of weak type (for unit size domains) $\|\chi\|_{L_{\infty}} \leqslant C h^{-\epsilon}\|\chi\|_{H^{1}}$ for $\chi \in S^{h}$, see Sections 7 and 8 for the technical details. We note that close to a concave corner the $L_{\infty}$ estimate derived via an $H_{1}$ estimate will be sharp.

In certain situations one can say more about the rate of convergence than what is given in (0.14) and (0.15). For example if $\beta_{j}<\min \left(r, 2 \beta_{M}\right)$, then $(0.15)$ predicts a higher rate of convergence in the interior than close to the $j$ th corner. In Theorem 5.1 we estimate precisely the rate of convergence at a point $x$ in terms of its distance $d$ to the vertex. For instance, if the maximal interior angle is concave $\left(\beta_{M}<1\right)$ then for $x$ close to $v_{M}$ we show, using $(0.16)$ and $(0.17)$, that with $\delta>0$ arbitrarily small, 


$$
\left|\left(u-u_{h}\right)(x)\right| \leqslant \begin{cases}C h^{2 \beta_{M}-\epsilon} d^{-\beta} M & \text { for } h^{1-\delta} \leqslant d \leqslant d_{0}, \\ C h^{\beta_{M}-\epsilon} & \text { for } d \leqslant h^{1-\delta} .\end{cases}
$$

Let us consider two consequences of (0.18). In Section 5 we show that if $\beta_{M}<$ 1 and the mesh is quasi-uniform of size $h$ near the vertex $v_{M}$, then for any $\delta>0$, the maximum error in $\left|u-u_{h}\right|$ over the domain $\Omega$ occurs at a point which is at most a distance $h^{1-\delta}$ away from the vertex $v_{M}$ (provided $k_{M}$ in (0.3) is not zero).

Our second application of $(0.18)$ concerns the problem of calculating what is sometimes called the stress intensity factor, i.e., the coefficient $k_{M}$ in the expansion (for $\left.\beta_{M}<1\right)$

$$
u(\rho, \theta)=k_{M} \rho^{\beta_{M}} \sin \left(\beta_{M} \theta\right)+O\left(\rho^{2 \beta} M\right)
$$

at the $M$ th vertex, see (6.1). In order to approximate $k_{M}$ we consider

$$
k_{M}(d, h)=u_{h}\left(d, \theta_{0}\right) / d^{\beta} M \sin \left(\beta_{M} \theta_{0}\right)
$$

where $0<\theta_{0}<\alpha_{M}$ is fixed.

The question then arises as to how $d$ should be chosen so that $k_{M}(d, h)$ is the best possible approximation to $k_{M}$. Now,

$$
\left|k_{M}-k_{M}(d, h)\right| \leqslant \frac{\left|u\left(d, \theta_{0}\right) \cdot-u_{h}\left(d, \theta_{0}\right)\right|}{\left|d^{\beta} M^{\prime} \sin \left(\beta_{M} \theta_{0}\right)\right|}+C d^{\beta_{M}} .
$$

Using (0.18), we make a choice that equalizes the error in the two terms above, namely $d=d_{h}=h^{2 / 3}$. We prove in Theorem 6.1 that

$$
\left|k_{M}-k_{M}\left(d_{h}, h\right)\right| \leqslant C h^{2 \beta_{M} / 3-\epsilon}, \quad \beta_{M}<1 .
$$

We also show, in Theorem 6.2, that the quantity $k_{M}\left(d_{h}, h\right) \rho^{\beta_{M}} \sin \left(\beta_{M} \theta\right)$ gives a potentially better approximation to $u$ than $u_{h}$ does, for $\rho \leqslant h^{2 / 3}$.

In Theorem 6.3 we use similar reasoning to exhibit how the next term in the expansion (0.19) (which is equal to $l_{M} \rho^{2 \beta_{M}} \sin \left(2 \beta_{M} \theta\right)$ if $3 \pi / 2<\alpha_{M}<2 \pi$ ) can be found approximately.

To conclude this introduction we give an outline of the rest of the paper. In Section 1 we collect notation and regularity results concerning the problem $(0.1)$ that will be needed. Section 2 lists assumptions on the finite element spaces. In Section 3 we state precisely the two basic results $(0.16)$ and $(0.17)$. The proofs of these are given in Sections 7 and 8 . Section 4 is concerned with proving $(0.14)$ and $(0.15)$. In Section 5 we give the proof of $(0.18)$ and calculate the location of the maximal error. In Section 6 the estimate $(0.18)$ is applied in connection with the question of approximate calculation of stress intensity factors. Finally, in Section 9 we give examples of finite element spaces.

1. Preliminaries. In this section we shall introduce notation and collect certain regularity results for the problem $(0.1)$.

In this paper $C, \epsilon$ and $\epsilon^{\prime}$ will denote positive constants, not necessarily the same at each occurrence, but independent of $h$. For simplicity in writing, we make the convention that $C$ may depend on $\epsilon$ and $\epsilon^{\prime}$. 
Throughout, $\Omega$ will be the fixed polygonal domain occurring in (0.1), cf. Remark 0.1 in the Introduction. We first set some terminology relating to subsets of $\Omega$.

For $D_{1} \subseteq D \subseteq \Omega$, define

$$
\operatorname{dist}_{y}\left(D_{1}, D\right)=\inf _{x \in \partial D_{1} \backslash\left(\partial D_{1} \cap \partial \Omega\right)} \operatorname{dist}(x, \partial D \backslash(\partial \Omega \cap \partial D))
$$

and let $D_{1} \$ D$ signify that $D_{1} \subseteq D$ with $\operatorname{dist}_{\Varangle}\left(D_{1}, D\right)>0$.

For $j$ a nonnegative integer and $1 \leqslant q \leqslant \infty, W_{q}^{j}(D)$ will be the usual Sobolev spaces with norms

$$
\|v\|_{w_{q}^{j}(D)}= \begin{cases}\left(\sum_{k<j}|v|_{w_{q}^{k}(D)}^{q}\right)^{1 / q}, & 1 \leqslant q<\infty, \\ \max _{k<j}|v|_{w_{\infty}^{k}(D)}, & q=\infty\end{cases}
$$

where

$$
|v|_{W_{q}^{k}(D)}= \begin{cases}\left(\sum_{|\alpha|=k}\left\|D^{\alpha} v\right\|_{L_{q}}^{q}\right)^{1 / q}, & 1 \leqslant q<\infty, \\ \max _{|\alpha|=k}\left\|D^{\alpha} v\right\|_{L_{\infty}(D)}, & q=\infty .\end{cases}
$$

In particular, for $q=2$ we shall write $H^{j}(D)$ for $w_{2}^{j}(D)$ and use the notation $\|v\|_{j, D}=$ $\|v\|_{w i}^{j(D)},|v|_{j, D}=|v|_{w_{2}^{j}(D)}$.

We shall need to extend the definition of the spaces $H^{j}(D)$ to nonintegral and negative values of $j$.

For $j$ positive and nonintegral, let $j=J+\sigma$, where $J$ is integral and $0<\sigma<1$. The norm in $H^{j}(D)$ is given by

where

$$
\|v\|_{j, D}=\|v\|_{H^{j}(D)}=\left(\|v\|_{J, D}^{2}+|v|_{j, D}^{2}\right)^{3 / 2}
$$

$$
|v|_{j, D}=\left(\sum_{|\alpha|=J}\left|D^{\alpha} v\right|_{\sigma, D}^{2}\right)^{1 / 2}
$$

with

$$
|v|_{\sigma, D}=\left(\int_{D} \int_{D} \frac{|v(x)-v(y)|^{2}}{|x-y|^{2 \sigma+2}} d x d y\right)^{1 / 2}
$$

For properties of these spaces on Lipschitz domains, cf., e.g. Grisvard [14], and Necas [22, Chapter 2], [23] where further references may be found. As general references we also note Lions and Magenes [20] and Slobodeckii [33].

We notice that for $D_{1} \subseteq D_{2}$,

$$
\|v\|_{j, D_{1}} \leqslant\|v\|_{j, D_{2}}, \quad j \geqslant 0
$$

and for $D_{1}$ and $D_{2}$ disjoint sets, 


$$
\|v\|_{j, D_{1}}^{2}+\|v\|_{j, D_{2}}^{2} \leqslant\|v\|_{j, D_{1} \cup D_{2}}^{2}, \quad j \geqslant 0 .
$$

For $j>1 / 2$ and $D \subseteq \Omega$ we define

$$
\overleftarrow{H}^{j}(D)=\left\{v \in H^{j}(D): v=0 \text { on } \partial D \cap \partial \Omega\right\} .
$$

In particular then,

$$
\stackrel{\leftrightarrow}{H}^{j}(\Omega)=\left\{\begin{array}{l}
\stackrel{\rho}{H}^{j}(\Omega), \text { closure of } \mathrm{C}_{0}^{\infty}(\Omega) \text { for } 1 / 2<j \leqslant 1, \\
H^{j}(\Omega) \cap \stackrel{\circ}{H}^{1}(\Omega) \text { for } j \geqslant 1 .
\end{array}\right.
$$

We shall also use the notation

$$
H^{j}(D)=\left\{v \in H^{j}(D): v \equiv 0 \text { in a neighborhood of } \partial D \backslash(\partial D \cap \partial \Omega)\right\} \text {. }
$$

Similarly, $\hat{C}^{\infty}(D)$ and $C^{\infty}(D)$ designate the subspaces of $C^{\infty}(D)$ of functions that vanish on $\partial D \cap \partial \Omega$, respectively in a neighborhood of $\partial D \backslash(\partial D \cap \partial \Omega)$.

For $j$ a negative number, we set

$$
\|v\|_{j, D}=\underset{\varphi \in C^{\infty}(D) \cap C^{\infty}(D)}{<} \frac{(v, \varphi)}{\|\varphi\|_{-j, D}}
$$

where $(v, \varphi)=\int_{D} v \varphi d x$. Similarly,

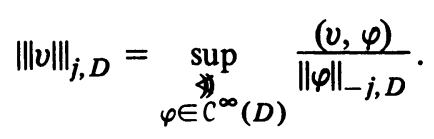

We have clearly

$$
\|v\|_{j, D} \leqslant\|v\|_{j, D}, \quad j<0 .
$$

For $j \in Z^{-}$, the negative integers, the following two properties hold:

If $D_{1} \subseteq D_{2}$, then

$$
\|v\|_{j, D_{1}} \leqslant\|v\|_{j, D_{2}}, \quad\|v\|_{j, D_{1}} \leqslant\|v\|_{j, D_{2}}, \quad j \in Z^{-} .
$$

For $D_{1}$ and $D_{2}$ disjoint sets,

$$
\left\{\begin{array}{l}
\|v\|_{j, D_{1}}^{2}+\|v\|_{j, D_{2}}^{2} \leqslant\|v\|_{j, D_{1} \cup D_{2}}^{2}, \\
\|v\|_{j, D_{1}}^{2}+\|v\|_{j, D_{2}}^{2} \leqslant\|v\|_{j, D_{1} \cup D_{2}}^{2}, \quad j \in Z^{-} .
\end{array}\right.
$$

For a proof of the last property, see [31, Lemma 1.1].

Let us also recall Sobolev's inequality, which we shall use in the following form.

For $D$ a Lipschitz domain,

$$
\|v\|_{w_{p}^{l}(D)} \leqslant C(D, p, l, q, k)\|v\|_{w_{q}^{k}(D)}, \quad q>\frac{2 p}{2+p(k-l)}, \quad k>l .
$$

In case $p$ (or $q$ ) is equal to 2 , then $l$ (or $k$ ) may assume nonintegral values. This result can be made to follow from Uspenskii [37] by use of a suitable extension operator, Stein [34]. See also Necas [22].

Finally, we shall collect a few results concerning the problem (0.1), 


$$
\begin{cases}-\Delta u=f & \text { in } \Omega, \\ u=0 & \text { on } \partial \Omega .\end{cases}
$$

Following Kellogg [17], [18] and other references given there we have:

Let $-1<s<\beta_{M}-1, \beta_{M}>1 / 2$. For any $\epsilon>0$ there exists a constant $C=$ $C(\epsilon, s)$ independent of $f$ such that

$$
\|u\|_{s+2-\epsilon, \Omega} \leqslant C\|f\|_{s, \Omega} .
$$

In fact, except when certain exceptional angles are present, the result holds with $\epsilon=0$.

If the right-hand side in (0.1) is sufficiently smooth we have the following results, Kellogg [17].

Let $s \geqslant 0$ be a number which does not belong to a certain set of exceptional values, i.e. the set

$$
\left\{n \beta_{j}-1, n=1,2, \ldots ; j=1, \ldots, M\right\} .
$$

Then for $f \in H^{s}(\Omega)$ we have

$$
u=\sum_{i=1}^{I} c_{i} S_{i}+w
$$

where:

(i) $w \in H^{s+2}(\Omega) \cap \stackrel{\circ}{H}^{1}(\Omega)$ and with $C$ independent of $f$,

$$
\|w\|_{s+2, \Omega} \leqslant C\|f\|_{s, \Omega},
$$

(ii) the functions $S_{j}$, the singular functions, are independent of $f$ and each $S_{j}$ may be taken to vanish outside of a neighborhood of one of the vertices. In a neighborhood of the $j$ th vertex one has upon introducing polar coordinates $(\rho, \theta)$, with $0 \leqslant \theta \leqslant \alpha_{j}$ lying in the interior of the wedge,

$$
u(\rho, \theta)=\sum_{0<m \beta_{j}<s+1} c_{j, m}(f) S_{j, m}(\rho, \theta)+w
$$

where for any $\epsilon>0$,

$$
\left|\left(D_{x, y}^{\alpha} S_{j, m}\right)(\rho, \theta)\right| \leqslant C \rho^{m \beta_{j}-|\alpha|-\epsilon} .
$$

In fact, unless $m \beta_{j}$ is an integer,

$$
S_{j, m}(\rho, \theta)=\rho^{m \beta_{j}} \sin \left(m \beta_{j} \theta\right),
$$

near the $j$ th vertex. In the case that $m \beta_{j}$ is integral, logarithmic terms may enter.

(iii) the coefficients $c_{i}=c_{i}(f)$ satisfy

$$
\left|c_{i}\right| \leqslant c\|f\|_{s, \Omega} .
$$

2. The Finite Element Spaces. In this section we collect the assumptions on the finite element spaces that will be used. Since we are concerned with the local behavior of the finite element solution, our hypotheses will be local in nature. Along with locally quasi-uniform meshes, certain non-quasi-uniform partitions will be allowed, cf. the examples given in Section 9. 
Let $S^{h}(\Omega), 0<h<1$, denote a one-parameter family of finite dimensional subspaces of $\stackrel{\circ}{H}^{1}(\Omega) \cap W_{\infty}^{1}(\Omega)$. In particular, the functions in $S^{h}(\Omega)$ vanish on $\partial \Omega$. For $D \subseteq \Omega$ we define $S^{h}(D)$ by restriction, and also set

$S^{h}(D)=\left\{\chi \in S^{h}(D): \chi\right.$ vanishes in a neighborhood of $\left.\partial D \backslash(\partial \Omega \cap \partial D)\right\}$.

We assume that there exists a constant $k_{0}$ such that the assumptions A.1-A.4 below are satisfied.

A.1. There exists an integer $r \geqslant 2$ and a constant $C_{1}$ such that the following holds:

Let $D_{1} \Varangle D$ with dist $\left(D_{1}, D\right) \geqslant k_{0} h$. Then for each $v \in \hat{H}^{1}(D) \cap W_{\infty}^{r}(D)$ there exists a $\chi \in S^{h}(D)$ such that

$$
\|v-\chi\|_{L_{\infty}\left(D_{1}\right)}+h\|v-\chi\|_{W_{\infty}^{1}\left(D_{1}\right)} \leqslant C_{1} h^{r}|v|_{W_{\infty}^{r}(D)} .
$$

Furthermore, if $v \in H^{1}\left(D_{1}\right)$, then $\chi \in S^{h}(D)$.

Our next assumption was introduced in [28] and is sometimes referred to as a "superapproximation" condition.

A.2. There exist constants $C_{2}$ and $l_{0}, l_{0}$ integer, such that the following holds:

Let $D_{1} \ggg D_{2} \ggg D$ with dist $\left(D_{1}, D_{2}\right) \geqslant k_{0} h$, and let $\omega \in C^{\infty}\left(D_{1}\right)$. Then for $\chi \in S^{h}(D)$ there exists an $\eta \in S^{h}(D)$ such that

$$
\|\omega \chi-\eta\|_{1, D} \leqslant C_{2} h\|\omega\|_{w_{\infty}^{l_{0}\left(D_{1}\right)}}\|\chi\|_{1, D_{2}} .
$$

We shall need a weak form of inverse properties.

A.3. There exists a number $\gamma \geqslant 1$ such that the following holds:

(i) There exists a constant $C_{3}$ such that if $D_{1} \$ D$ with dist $\left(D_{1}, D\right) \geqslant k_{0} h$, then for $\chi \in S^{h}(D)$,

$$
\|\chi\|_{L_{\infty}\left(D_{1}\right)} \leqslant C_{3} h^{-\gamma 2 / q}\|\chi\|_{L_{q}(D)} \text { for } 2 \leqslant q<\infty .
$$

(ii) Let $p$ be a nonnegative integer. There exists a constant $C_{4}=C_{4}(p)$ such that if $D_{1} \ggg D$ with dist $\left.D_{1}, D\right) \geqslant k_{0} h$, then for $\chi \in S^{h}(D)$,

$$
\|\chi\|_{1, D_{1}} \leqslant C_{4} h^{-\gamma(p+1)}\|\chi\|_{-p, D}
$$

Lastly, we shall make the assumption that if the intersection of a disc of radius $d$ with $\Omega$ is transformed via a similarity transformation to a domain of unit size, then the transformed finite element space satisfies A.1, A.2 and a slightly modified form of A.3 with $h$ replaced by $h / d$.

Let $D_{d}$ be the intersection with $\Omega$ of a disc of radius $d$ centered at $x_{0} \in \bar{\Omega}$. The linear transformation $y=\left(x-x_{0}\right) / d$ takes $D_{d}$ into $\widetilde{D}$ and $S^{h}\left(D_{d}\right)$ into a new function space $S(\widetilde{D})$.

A.4. Given any $\delta>0$, if $1 \geqslant d \geqslant h^{1-\delta}$, then $S(\widetilde{D})$ satisfies A.1 and A.2 with $h$ replaced by $h / d$, and satisfies A.3 with $h$ replaced by $h / d$ and $\gamma$ replaced by $\gamma_{\delta}=$ $1+(\gamma-1) / \delta$. The rest of the constants remain the same.

We remark that in our examples, A.1 and A.2 will be satisfied if $d \geqslant k_{0} h$, and 
if furthermore the mesh is quasi-uniform, then A.3 will be true with $\gamma=1$ for $d \geqslant$ $k_{0} h$.

In our subsequent work we shall frequently need certain consequences of the approximation hypothesis A.1. The first result, Lemma 2.1 below, concerns approximation in $L_{2}$ based norms, and approximation of functions with low regularity. In many examples, a natural interpolant could be utilized to show these results directly, but in general one has to smooth the function before applying the interpolant, cf. Hilbert [15] and also Clement [9], Strang [35]. In our case we also want to smooth so as to preserve the vanishing of the functions on $\partial \Omega$. A suitable smoothing operator was given in Nitsche [26]. For completeness we shall exhibit its form near a corner. Here the corner is normalized so that the sides are along the negative $x$ and $y$ axes for the concave case, or positive $x$ and $y$ axes for the convex case. By use of a linear transformation, it suffices to treat these two cases. With $\kappa(\cdot)$ and $\eta(\cdot)$ having suitable properties, see [26], we set

$$
\begin{aligned}
S v(x, y)= & \int_{0}^{1} \kappa(r)\left[\int_{0}^{1} \kappa(s) v(x+r h, y+s h) d s-\eta\left(\frac{y}{h}\right) \int_{0}^{1} \kappa(s) v(x+r h, s h) d s\right] d r \\
& -\eta\left(\frac{x}{h}\right) \int_{0}^{1} \kappa(r)\left[\int_{0}^{1} \kappa(s) v(r h, y+s h) d s-\eta\left(\frac{y}{h}\right) \int_{0}^{1} \kappa(s) v(r h, s h) d s\right] d r .
\end{aligned}
$$

Using this smoothing operator and A.1 one may prove the following result.

Lемма 2.1. There exists a constant $C$ such that the following holds:

Let $D_{1} \ggg D$ with $\operatorname{dist}_{\oiint}\left(D_{1}, D\right) \geqslant\left(k_{0}+1\right) h$. Then for each $v \in \stackrel{K}{H}^{1}(D) \cap W_{\infty}^{r}(D)$ there exists a function $\chi \in S^{h}(D)$ such that

$$
\|v-\chi\|_{0, D_{1}}+h\|v-\chi\|_{1, D_{1}} \leqslant C h^{r}\|v\|_{r, D}
$$

and

$$
\|v-\chi\|_{L_{\infty}\left(D_{1}\right)}+h\|v-\chi\|_{w_{\infty}^{1}\left(D_{1}\right)} \leqslant C h^{r}\|v\|_{w_{\infty}^{r}(D)}
$$

Furthermore, for each $v \in \overleftarrow{H}^{1}(D) \cap H^{2}(D)$ there exists a function $\chi \in S^{h}(D)$ such that

$$
\|v-\chi\|_{0, D_{1}}+h\|v-\chi\|_{1, D_{1}} \leqslant C h^{2}\|v\|_{2, D}
$$

The corresponding results hold after a homothety by $1 / d$, cf. A.4, with $h$ replaced by $h / d$ for $d \geqslant h^{1-\delta}$; the constants occurring remain the same.

Finally, we shall need a global approximation result in Sobolev spaces with nonintegral smoothness parameters. The proof will be given in Appendix 2 .

LemmA 2.2. Assume that A.1 holds, and let $s$ be a real number, $1 \leqslant s \leqslant r$. There exists a constant $C$ such that the following holds.

For any $v \in \stackrel{\circ}{H}^{1}(\Omega) \cap H^{s}(\Omega)$ there exists a function $\chi \in S^{h}(\Omega)$ such that

$$
\|v-\chi\|_{1, \Omega} \leqslant C h^{s-1}\|v\|_{s, \Omega}
$$


3. Two Basic Results. In this section we state our two main theorems. The investigations in Sections 4-6 will be based on those theorems, the proofs of which will be given later in Sections 7 and 8, respectively.

In our first result we extend, under our present more general hypotheses, the interior $H^{1}$ estimates of [27], and give estimates up to the boundary. The resulting bound includes the case when the domains are small. On unit size domains, the $H^{1}$ estimate is converted into an estimate in the maximum norm using the following simple device: Given $\epsilon>0$, let $q$ be such that $\epsilon=2 \gamma / q$. Then by A.3 and Sobolev's inequality with $D_{1} \ggg D$,

$$
\|\chi\|_{L_{\infty}\left(D_{1}\right)} \leqslant C h^{-\epsilon}\|\chi\|_{L_{q}(D)} \leqslant C h^{-\epsilon}\|\chi\|_{1, D} \quad \text { for } \chi \in S^{h} .
$$

This result will be particularly useful later on in discussing the error in the neighborhood of a concave corner. Let us remark that if the mesh is quasi-uniform then the factor $h^{-\epsilon}$ may be replaced by $\ln / h$.

TheOREM 3.1. Assume that A.1, A.2, A.3 and A.4 hold. Let $p$ be a nonnegative integer, and $0<\delta \leqslant 1,0<\epsilon \leqslant 1$. There exist constants $C=C(p, \delta, \epsilon)$ and $h_{1}=$ $h_{1}(p, \delta)>0$ such that the following holds:

Let $D_{1} \Varangle D$ with dist $_{\Varangle}\left(D_{1}, D\right)=d \geqslant h^{1-\delta}$, and let $u \in \stackrel{K}{H}^{1}(D)$ and $u_{h} \in S^{h}(D)$ be such that

$$
A\left(u-u_{h}, \varphi\right)=0 \text { for all } \varphi \in S^{h}(D) \text {. }
$$

Then for $h \leqslant h_{1}$ we have for any $\chi \in S^{h}(D)$,

$$
\begin{aligned}
\left\|u-u_{h}\right\|_{L_{\infty}\left(D_{1}\right)} \leqslant C h^{-\epsilon}\left\{\|u-\chi\|_{L_{\infty}(D)}+d^{-1}\|u-\chi\|_{0, D}\right. \\
\left.+|u-\chi|_{1, D}+d^{-1-p}\left\|u-u_{h} \mid\right\|_{-p, D}\right\}
\end{aligned}
$$

We remark that in the proof of this result we do not use the hypothesis A.1 in itself; only the consequence (2.7) of Lemma 2.1 is employed.

Our second result is a generalization of the interior maximum norm estimates of [31], given there for quasi-uniform meshes.

THEOREM 3.2. Assume that A.1, A.2, A.3 and A.4 hold. Let $p$ be a nonnegative integer, and $0<\delta \leqslant 1,0<\epsilon \leqslant 1$. There exist constants $C=C(p, \delta, \epsilon)$ and $h_{2}=$ $h_{2}(p, \delta, \epsilon)>0$ such that the following holds.

Let $D_{1} \ggg D$ with dist $\left(D_{1}, D\right)=d \geqslant h^{1-\delta}$, and assume that $D$ contains no concave vertex of $\Omega$. Let $u \in H^{1}(D) \cap W_{\infty}^{1}(D)$ and $u_{h} \in S^{h}(D)$ satisfy

$$
A\left(u-u_{h}, \varphi\right)=0 \text { for all } \varphi \in S^{h}(D) \text {. }
$$

Then for $h \leqslant h_{2}$ we have for any $\chi \in S^{h}(D)$,

$$
\begin{aligned}
& \left\|u-u_{h}\right\|_{L_{\infty}\left(D_{1}\right)} \\
& \quad \leqslant C h^{-\epsilon}\left(h|u-\chi|_{W_{\infty}^{1}(D)}+\|u-\chi\|_{L_{\infty}(D)}+d^{-1-p}\left\|u-u_{h}\right\|_{-p, D}\right) .
\end{aligned}
$$

Let us again remark that in the proof of this theorem, A.1 is used only via its consequence (2.7) of Lemma 2.1. 
4. The Dirichlet Problem on a Polygonal Domain. Let $\Omega$ be the polygonal domain in (0.1) with interior angles $0<\alpha_{1} \leqslant \cdots \leqslant \alpha_{M}<2 \pi$, and set $\beta_{j}=\pi / \alpha_{j}$. Let $\Omega_{j} \ggg \Omega, j=1, \ldots, M$, be fixed domains which contain only the $j$ th corner, and set $\Omega_{0}=\Omega \backslash\left(\bigcup_{j=1}^{M} \bar{\Omega}_{j}\right)$.

Let $u \in \stackrel{\circ}{H}^{1}(\Omega)$ be the solution of $(0.1)$ where for simplicity we assume that $f \in$ $C^{\infty}(\bar{\Omega})$, and let $u_{h} \in S^{h}(\Omega)$ be the finite element solution given by $(0.2)$. In the above notation we have the following result concerning local rate of convergence in the maximum norm.

Theorem 4.1. Assume that A.1, A.2, A.3 and A.4 hold. Let $\epsilon>0$ be given. There exists a constant $C=C(f, \epsilon)$ such that for $h$ sufficiently small,

$$
\left\|u-u_{h}\right\|_{L_{\infty}\left(\Omega_{0}\right)} \leqslant C h^{\min \left(r, 2 \beta_{M}\right)-\epsilon},
$$

and

$$
\left\|u-u_{h}\right\|_{L_{\infty}\left(\Omega_{j}\right)} \leqslant C h^{\min \left(r, \beta_{j}, 2 \beta_{M}\right)-\epsilon} \text { for } j=1, \ldots, M .
$$

Proof. Let $\Omega_{j} \ggg \Omega_{j}^{\prime}$ with $\Omega_{0}^{\prime}$ containing no corner, and $\Omega_{j}^{\prime}, j=1, \ldots, M$, containing only the $j$ th vertex. We shall use the following two lemmas. The first concerns local approximation of solutions of (0.1).

Lemma 4.1. Assume that A.1 holds.

(i) There exists a function $\chi \in S^{h}\left(\Omega_{0}^{\prime}\right)$ such that

$$
\|u-\chi\|_{L_{\infty}\left(\Omega_{0}^{\prime}\right)}+h|u-\chi|_{w_{\infty}^{1}\left(\Omega_{0}^{\prime}\right)} \leqslant C(f) h^{r} .
$$

(ii) Let $j \geqslant 1$ be such that $\beta_{j}>1$ (convex corner). Given $\epsilon^{\prime}>0$ there exists a function $\chi \in S^{h}\left(\Omega_{j}^{\prime}\right)$ such that

$$
\|u-\chi\|_{L_{\infty}\left(\Omega_{j}^{\prime}\right)}+h|u-\chi|_{W_{\infty}^{1}\left(\Omega_{j}^{\prime}\right)} \leqslant C\left(f, \epsilon^{\prime}\right) h^{\min \left(r, \beta_{j}\right)-\epsilon^{\prime}} .
$$

(iii) Let $j \geqslant 1$ be such that $\beta_{j}<1$ (concave corner). Given $\epsilon^{\prime}>0$ there exists a function $\chi \in S^{h}\left(\Omega_{j}^{\prime}\right)$ such that

$$
\|u-\chi\|_{L_{\infty}\left(\Omega_{j}^{\prime}\right)}+\|u-\chi\|_{1, \Omega_{j}^{\prime}} \leqslant C\left(f, \epsilon^{\prime}\right) h^{\beta_{j}-\epsilon^{\prime}} .
$$

Our next lemma may be of some independent interest.

Lemma 4.2. Assume that A.1 holds. (Only (2.8) of Lemma 2.2 will be used in the proof.) Given $\epsilon^{\prime}>0$ we have

$$
\left\|u-u_{h}\right\|_{1-\beta_{M}-\epsilon^{\prime}, \Omega} \leqslant C\left(f, \epsilon^{\prime}\right) h^{\min \left(2(r-1), 2 \beta_{M}\right)-2 \epsilon^{\prime}}, \quad \beta_{M}>1,
$$

and

$$
\left\|u-u_{h}\right\|_{1-\beta_{M}-\epsilon^{\prime}, \Omega} \leqslant C\left(f, \epsilon^{\prime}\right) h^{2 \beta_{M}-2 \epsilon^{\prime}}, \quad 1 / 2<\beta_{M}<1-\epsilon^{\prime} .
$$

Assuming for the moment that Lemmas 4.1 and 4.2 hold, we shall complete the proof of Theorem 4.1. Let us first treat the case of a concave angle $\alpha_{j}$, i.e., $1 / 2<\beta_{j}<1$. By Theorem 3.1, with $d=1$, we have

$$
\left\|u-u_{h}\right\|_{L_{\infty}\left(\Omega_{j}\right)} \leqslant C h^{-\epsilon^{\prime}}\left(\|u-\chi\|_{L_{\infty}\left(\Omega_{j}^{\prime}\right)}+\|u-\chi\|_{1, \Omega_{j}^{\prime}}+\left\|u-u_{h}\right\|_{0, \Omega}\right) \text {. }
$$

The desired result follows from (4.5) and (4.7). 
For the case of $\alpha_{j}$ convex, i.e., $\beta_{j}>1$, we use Theorem 3.2 to obtain for any integer $p$,

$$
\begin{aligned}
& \left\|u-u_{h}\right\|_{L_{\infty}\left(\Omega_{j}\right)} \\
& \quad \leqslant C h^{-\epsilon^{\prime}}\left(h|u-\chi|_{w_{\infty}^{1}\left(\Omega_{j}^{\prime}\right)}+\|u-\chi\|_{L_{\infty}\left(\Omega_{j}^{\prime}\right)}+\left\|u-u_{h}\right\|_{-p, \Omega_{j}^{\prime}}\right) .
\end{aligned}
$$

The first two terms on the right are now taken care of by (4.4). For the last term, we may replace it by $\left\|u-u_{h}\right\|_{-p, \Omega}$ (see (1.4)), and if $p>\beta_{M}-1$ we can utilize Lemma 5.2 to conclude the proof.

The proof for the case of $\Omega_{0}$ is similar and follows using Theorem 3.2, (4.3) and Lemma 4.2.

This proves Theorem 4.1 .

It remains to prove the two lemmas above.

Proof of Lemma 4.1. We shall give the details for (4.5); the other cases are similar. Note that (4.5) demands simultaneous approximation in $H^{1}$ and $L_{\infty}$. The proof is based on using the approximation results (2.5), (2.6) of Lemma 2.1, and the detailed information concerning the behavior of $u$ that is contained in (1.8)-(1.13). First, by (1.8) we may write $u=s+w$ near the corner, where $w \in \stackrel{\circ}{H}^{1}(\Omega) \cap W_{\infty}^{r}(\Omega)$. By Lemma $2.1, w$ can be approximated in the desired way.

For the singular part $s$, we proceed as follows. Let $\omega \in C^{\infty}\left(\Omega_{j}^{\prime}\right)$ be such that $\omega(x)=0$ if $\operatorname{dist}\left(v_{j}, x\right) \leqslant\left(k_{0}+1\right) h, \omega \equiv 1$ if $\operatorname{dist}\left(v_{j}, x\right) \geqslant 2\left(k_{0}+1\right) h$. Furthermore assume that

$$
\left\|D^{\alpha} \omega\right\|_{L_{\infty}\left(\Omega_{j}^{\prime}\right)} \leqslant C h^{-|\alpha|} \text {. }
$$

We now approximate $\omega s$ as in (2.5), (2.6), and using (1.11) and (1.13) we easily deduce the estimates sought. The proof is completed by noting (again using (1.11) and (1.13)) that

$$
\|\omega s-s\|_{L_{\infty}\left(\Omega_{j}^{\prime}\right)}+\|\omega s-s\|_{1, \Omega_{j}^{\prime}} \leqslant C h^{\beta_{j}-\epsilon^{\prime}} .
$$

This proves Lemma 4.1 .

We remark that it suffices to have $f$ in $H^{r-1+\epsilon}(\Omega)$ for the above argument.

Proof of Lemma 4.2. Let us first treat the case $\beta_{M}>1$. We have

$$
\left\|u-u_{h}\right\|_{1-\beta_{M^{-}} \epsilon^{\prime}, \Omega}=\sup _{\varphi \in C^{\infty}(\Omega)} \frac{\left(u-u_{h}, \varphi\right)}{\|\varphi\|_{\beta_{M^{-1}}+\epsilon^{\prime}, \Omega}} .
$$

For fixed $\varphi$, let $\psi$ be the solution of $-\Delta \psi=\varphi$ in $\Omega, \psi=0$ on $\partial \Omega$. Then

$$
\left(u-u_{h}, \varphi\right)=A\left(u-u_{h}, \psi\right)=A\left(u-u_{h}, \psi-\chi\right)
$$

for any $\chi \in S^{h}(\Omega)$. Thus,

$$
\left(u-u_{h}, \varphi\right) \leqslant\left\|u-u_{h}\right\|_{1, \Omega}\|\psi-\chi\|_{1, \Omega},
$$

and using Lemma 2.2 we may choose $\chi$ such that

$$
\|\psi-\chi\|_{1, \Omega} \leqslant C h^{\min (r-1, \beta} M^{\left.-\epsilon^{\prime}\right)}\|\psi\|_{1+\beta_{M^{-}} \epsilon^{\prime}, \Omega} .
$$

From the a priori estimate (1.7) we have 


$$
\|\psi\|_{1+\beta_{M^{-}} \epsilon^{\prime}, \Omega} \leqslant C\|\varphi\|_{\beta_{M^{-1}}+\epsilon^{\prime}, \Omega}
$$

and hence

$$
\left\|u-u_{h}\right\|_{1-\beta_{M}-\epsilon^{\prime}, \Omega} \leqslant C h^{\min \left(r-1, \beta_{M}-\epsilon^{\prime}\right)}\left\|u-u_{h}\right\|_{1, \Omega} .
$$

Similarly

$$
\left\|u-u_{h}\right\|_{1, \Omega} \leqslant C \inf _{x \in S^{h}(\Omega)}\|u-\chi\|_{1, \Omega} \leqslant C\left(f, \epsilon^{\prime}\right) h^{\min \left(r-1, \beta_{M^{-}} \epsilon^{\prime}\right)},
$$

and we obtain the desired result in the case of $\beta_{M}>1$.

In order to treat the case $1 / 2<\beta_{M}<1$ we shall need the following lemma:

LEMmA 4.3. Let $0<j<1 / 2$. There exists a constant $C$ such that

$$
\|v\|_{j, \Omega} \leqslant C \sup _{\varphi \in C^{\infty}(\Omega)} \frac{(v, \varphi)}{\|\varphi\|_{-j, \Omega}} .
$$

From this we have that

$$
\left\|u-u_{h}\right\|_{1-\beta_{M}-\epsilon^{\prime}, \Omega} \leqslant C \sup _{\varphi \in C^{\infty}(\Omega)} \frac{\left(u-u_{h}, \varphi\right)}{\|\varphi\|_{\beta_{M^{-1}}+\epsilon^{\prime}, \Omega}}
$$

and the proof of Lemma 4.2 then proceeds word for word as in the previous case.

It remains to prove Lemma 4.3.

Proof. We first note that after extension of $v$ by zero,

$$
\|v\|_{j, \Omega} \leqslant\|v\|_{j, R^{2}} \text {. }
$$

Using the equivalent norm $N_{j}(v)=\left(\int\left(1+|\xi|^{2}\right)^{j}|\hat{v}(\xi)|^{2} d \xi\right)^{1 / 2}$ on $H^{j}\left(R^{2}\right)$ in terms of the Fourier transform (cf. Slobodeckii [33], Neðas [22]) we find that

$$
\|v\|_{j, R^{2}} \leqslant C \sup _{\psi \in \mathrm{C}_{0}^{\infty}\left(R^{2}\right)} \frac{(v, \psi)}{\|\psi\|_{-j, R^{2}}} .
$$

We shall next establish that for $\psi \in C_{0}^{\infty}\left(R^{2}\right)$,

$$
\|\psi\|_{-j, \Omega} \leqslant C\|\psi\|_{-j, R^{2}}
$$

Combining this with (4.10) and (4.9) gives the result that

$$
\|v\|_{j, \Omega} \leqslant C \sup _{\psi \in C_{0}^{\infty}\left(R^{2}\right)} \frac{(v, \psi)}{\|\psi\|_{-j, \Omega}}
$$

which clearly implies the desired estimate (4.8).

It remains to verify (4.11). By definition we have

$$
\|\psi\|_{-j, \Omega}=\sup _{w \in C^{\infty}(\Omega) ; w=0 \text { on } \partial \Omega} \frac{(\psi, w)}{\|w\|_{j, \Omega}}
$$

From Nečas [23] we know that extending $w$ by zero from the Lipschitz domain $\Omega$ to $R^{2}$ is a continuous operation from $H^{j}(\Omega)$ to $H^{j}\left(R^{2}\right)$ for $0 \leqslant j<1 / 2$, i.e.,

$$
\|w\|_{j, R^{2}} \leqslant C\|w\|_{j, \Omega}
$$


Thus,

$$
\|\psi\|_{-j, \Omega} \leqslant C \sup _{w \in C^{\infty}(\Omega) ; w=0 \text { on } \partial \Omega} \frac{(\psi, w)}{\|w\|_{j, R^{2}}} \leqslant C\|\psi\|_{-j, R^{2}} .
$$

This proves (4.11) and completes the proof of the lemma.

5. Location of the Maximal Error in the Dirichlet Problem. In this section we shall consider meshes that have an element of "size $h$ " near the vertex of a concave maximal angle. We prove then that the maximal error in $u-u_{h}$ occurs within an $O\left(h^{1-\epsilon}\right)$ distance from that vertex.

If for some $j, \beta_{j}<\min \left(r, 2 \beta_{M}\right)$ then Theorem 4.1 predicts a higher rate of convergence in $\Omega_{0}$ than in $\Omega_{j}$. This suggests that in this instance the rate of convergence is an increasing function of the distance to the vertex. We shall prove that such is the case in a special but important situation. Other examples can be treated similarly.

Let

$$
\Omega_{j}^{d}=\left\{x: d / 2<\left|x-v_{j}\right|<d\right\} \cap \Omega
$$

where $d \leqslant d_{0}$ and $\Omega_{j}^{2 d}$ contains no other vertex of $\Omega$.

TheOREM 5.1. Assume that A.1, A.2, A.3 and A.4 hold. Furthermore, let

$$
\beta_{j} \leqslant 2 \beta_{M} \leqslant r .
$$

Given $0<\epsilon \leqslant 1$ and $0<\delta \leqslant 1$ there exists a constant $C$ such that for $h$ sufficiently small,

$$
\left\|u-u_{h}\right\|_{L_{\infty}\left(\Omega_{j}^{d}\right)} \leqslant\left\{\begin{array}{l}
C h^{2 \beta_{M^{-}} \epsilon} d^{-\beta} \text { for } h^{\left(2 \beta_{M^{-}} \beta_{j}\right) / \beta_{M}-\delta} \leqslant d \leqslant d_{0}, \\
C h^{\beta_{j}^{-\epsilon}} \text { for } d \leqslant h^{\left(2 \beta_{M^{-}} \beta_{j}\right) / \beta_{M^{-\delta}}} .
\end{array}\right.
$$

Note that the hypotheses in (5.1) are always fulfilled if $j=M$ and $\beta_{M}<1$. In this case we have

$$
\left\|u-u_{h}\right\|_{L_{\infty}\left(\Omega_{M}^{d}\right)} \leqslant\left\{\begin{array}{l}
C h^{2 \beta_{M}-\epsilon} d^{-\beta} \quad \text { for } h^{1-\delta} \leqslant d \leqslant d_{0}, \\
C h^{\beta_{M}-\epsilon} \text { for } d \leqslant h^{1-\delta} .
\end{array}\right.
$$

Proof. Let $\widetilde{\Omega}_{j}^{d}=\Omega_{j}^{d / 2} \cup \bar{\Omega}_{j}^{d} \cup \Omega_{j}^{2 d}$. From Theorem 3.2 we have that for $d \geqslant h^{1-\delta}$,

$$
\begin{aligned}
\left\|u-u_{h}\right\|_{L_{\infty}\left(\Omega_{j}^{d}\right)} \leqslant C h^{-\epsilon / 2}\left(h|u-\chi|_{W_{\infty}\left(\widetilde{\Omega}_{j}^{d}\right)}+\|u-\chi\|_{L_{\infty}\left(\widetilde{\Omega}_{j}^{d}\right)}\right. & \\
& +d^{-1-\left[\beta_{M}\right]}\left\|u-u_{h}\right\| \|_{\left.-\left[\beta_{M}\right], \widetilde{\Omega}_{j}^{d}\right),}
\end{aligned}
$$

where $\left[\beta_{M}\right]$ designates the integer part of $\beta_{M}$. By Lemma 2.1 and (1.8)-(1.13) we may assume that

$$
h|u-\chi|_{w_{\infty}^{1}\left(\widetilde{\Omega}_{j}^{d}\right)}+\|u-\chi\|_{L_{\infty}\left(\widetilde{\Omega}_{j}^{d}\right)} \leqslant C h^{r} d^{\beta_{j}-r-\epsilon / 2} .
$$

To complete the proof we use the following lemma. 
LEMMA 5.1. Under the above hypotheses, we have for any $\epsilon>0$,

$$
d^{-1-\left[\beta_{M}\right]}\left\|u-u_{h}\right\|_{-\left[\beta_{M}\right], \widetilde{\Omega}_{j}^{d}} \leqslant C d^{-\beta_{M} h^{2 \beta} M^{-\epsilon / 2}} .
$$

Assuming Lemma 5.1, we have from the above

$$
\left\|u-u_{h}\right\|_{L_{\infty}\left(\Omega_{j}^{d}\right)} \leqslant C h^{r} d^{\beta} j^{-r-\epsilon}+C d^{-\beta_{M} h^{2 \beta} M^{-\epsilon}} \text { for } h^{1-\delta} \leqslant d \leqslant d_{0} .
$$

Hence using (5.1),

$$
\left\|u-u_{h}\right\|_{L_{\infty}\left(\Omega_{j}^{d}\right)} \leqslant C d^{-\beta} M_{h}^{2 \beta_{M}-\epsilon} \text { for } h^{1-\delta} \leqslant d \leqslant d_{0} .
$$

From Theorem 4.1, $\left\|u-u_{h}\right\|_{L_{\infty}\left(\Omega_{j}^{d}\right)} \leqslant C h^{\beta_{j}-\epsilon}$ and we obtain the desired estimate (5.2).

It remains to prove Lemma 5.1.

Proof of Lemma 5.1. We have

$$
\left\|u-u_{h}\right\| \|_{-\left[\beta_{M}\right], \widetilde{\Omega}_{j}^{d}}=\sup _{\varphi \in C^{\infty}\left(\widetilde{\Omega}_{j}^{d}\right)} \frac{\left(u-u_{h}, \varphi\right)}{\|\varphi\|_{\left[\beta_{M}\right], \widetilde{\Omega}_{j}^{d}}} .
$$

For fixed $\varphi$, let $-\Delta \psi=\varphi$ in $\Omega, \psi=0$ on $\partial \Omega$. Then for any $\chi \in S^{h}(\Omega)$,

$$
\left(u-u_{h}, \varphi\right)=A\left(u-u_{h}, \psi-\chi\right) .
$$

Thus, by use of Lemma 2.2 (cf. the proof of Lemma 4.2),

$$
\begin{aligned}
\left|\left(u-u_{h}, \varphi\right)\right| & \leqslant C\left\|u-u_{h}\right\|_{1, \Omega}\|\psi-\chi\|_{1, \Omega} \\
& \leqslant C h^{2 \beta_{M}-2 \epsilon^{\prime}}\|\psi\|_{1+\beta_{M^{-}} \epsilon^{\prime}, \Omega} \quad \text { for any } \epsilon^{\prime}>0
\end{aligned}
$$

From (1.7) we also have

$$
\|\psi\|_{1+\beta_{M^{-}} \epsilon^{\prime}, \Omega} \leqslant C\|\varphi\|_{\beta_{M^{-1}-\epsilon^{\prime} / 2, \Omega}}
$$

We now separate the cases $\beta_{M}>1$ and $\beta_{M}<1$. When $\beta_{M}>1$ we have by Sobolev's inequality (1.6), with $\epsilon^{\prime}$ small,

$$
\|\varphi\|_{\beta_{M^{-1}-\epsilon^{\prime} / 2, \Omega}} \leqslant C\|\varphi\|_{w_{q}^{\left[\beta_{M}\right]_{(\Omega)}}}
$$

where $q=2 /\left(2+\left[\beta_{M}\right]-\beta_{M}\right)$. Note now that the support properties of $\varphi$ give

$$
\|\varphi\|_{w_{q}^{\left[\beta_{M}\right]}(\Omega)} \leqslant C d^{1+\left[\beta_{M}\right]-\beta_{M}}\|\varphi\|_{\left[\beta_{M}\right], \widetilde{\Omega}_{j}^{d}} .
$$

Thus, by (5.5)-(5.9) we obtain for $\beta_{M}>1$,

$$
\left\|u-u_{h}\right\|_{-\left[\beta_{M}\right], \widetilde{\Omega}_{j}^{d}} \leqslant C h^{2 \beta_{M}-2 \epsilon^{\prime}} d^{1+\left[\beta_{M}\right]-\beta_{M}}
$$

and the desired estimate (5.4) follows in this case if $\epsilon^{\prime}$ is taken small enough.

For the situation when $\beta_{M}<1$, i.e. $\left[\beta_{M}\right]=0$, proceed as above up to the point (5.7). Write then

$$
\|\varphi\|_{\beta_{M^{-1}}-\epsilon^{\prime} / 2, \Omega} \leqslant \sup _{w \in C^{\infty}(\Omega)} \frac{(\varphi, w)}{\|w\|_{1-\beta_{M^{+}}+\epsilon^{\prime} / 2, \Omega}}
$$


Here,

$$
(\varphi, w) \leqslant\|\varphi\|_{0, \widetilde{\Omega}_{j}^{d}}\|w\|_{0, \widetilde{\Omega}_{j}^{d}}
$$

and by use of Sobolev's inequality (1.6),

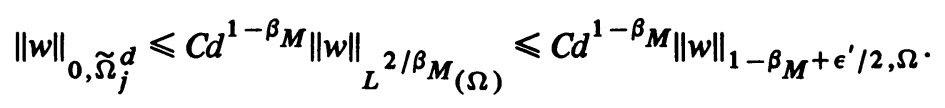

The desired result follows in this case too.

This completes the proof of Lemma 5.1.

We shall now discuss the location of the maximal error. For simplicity, we shall restrict ourselves to the case of a concave maximal angle which is strictly larger than the other angles.

In a neighborhood of the vertex $v_{M}$ let

$$
u(\rho, \theta)=k_{M} \rho^{\beta} \sin \left(\beta_{M} \theta\right)+\cdots
$$

with $k_{M} \neq 0$. We assume that there exists a constant $C>0$ such that for $h$ sufficiently small,

$$
C h^{\beta} M \leqslant \inf _{\chi \in S^{h}(\Omega)}\|u-\chi\|_{L_{\infty}(\Omega)}
$$

This would, e.g. be the case in our examples of Section 9 if elements of size $h$ occurred at the vertex $v_{M}$. For, the problem of then showing (5.10) is easily reduced to the one dimensional problem of approximating $k x^{\beta} M, k>0$, on $(0, h)$, by polynomials $\pi$ of a certain degree $t \geqslant 1$. We have

$$
\left\|k x^{\beta} M-\pi\right\|_{L_{\infty}(0, h)}=k h^{\beta}\left\|_{\| y^{\beta} M}-\tilde{\pi}(y)\right\|_{L_{\infty}(0,1)},
$$

where $y=x / h, \tilde{\pi}(y)=k^{-1} h^{-\beta_{M}} \pi(h y)$. Since with $P_{t}$ the polynomials on $(0,1)$ of degree $t$,

$$
\inf _{\pi \in P_{t}}\left\|y^{\beta_{M}}-\tilde{\pi}(y)\right\|_{L_{\infty}(0,1)} \geqslant c>0 \quad\left(\beta_{M}<1\right)
$$

we obtain

$$
\inf _{\pi}\left\|k x^{\beta} M-\pi\right\|_{L_{\infty}(0, h)} \geqslant c k h^{\beta} M
$$

This proves (5.10).

Theorem 5.2. Let A.1, A.2, A.3, A.4 and (5.10) hold. Assume that $\beta_{M}<1$ and $\beta_{M}<\beta_{M-1}$. Let $\bar{x}$ be such that $\left\|u-u_{h}\right\|_{L_{\infty}(\Omega)}=\left|\left(u-u_{h}\right)(\bar{x})\right|$ and let $0<\delta<$ $\left(\beta_{M-1}-\beta_{M}\right)$ be given. Then for $h$ sufficiently small,

$$
\left|\bar{x}-v_{M}\right| \leqslant h^{1-\delta} .
$$

Proof. Let $\epsilon>0$ be sufficiently small. Then from Theorem 4.1 we have that outside of $\Omega_{M}$,

$$
\left\|u-u_{h}\right\|_{L_{\infty}\left(\Omega \backslash \Omega_{M}\right)} \leqslant C h^{\min \left(r, \beta_{M-1}, 2 \beta_{M}\right)-\epsilon} \leqslant C h^{\beta_{M}+\delta / 2} .
$$


From (5.3) of Theorem 5.1 we see that for $d \geqslant h^{1-\delta}$,

$$
\left\|u-u_{h}\right\|_{L_{\infty}\left(\Omega_{M}^{d}\right)} \leqslant C h^{2 \beta_{M}^{-\epsilon}} d^{-\beta} \leqslant C h^{\beta} M^{+\delta / 4} .
$$

Thus for $\left|x-v_{M}\right| \geqslant h^{1-\delta}$,

$$
\left|\left(u-u_{h}\right)(x)\right| \leqslant C h^{\beta} M^{+\delta / 4}
$$

and since by assumption

$$
\left|\left(u-u_{h}\right)(\bar{x})\right| \geqslant C h^{\beta} M,
$$

we obtain the estimate (5.11). This proves the theorem.

6. Calculation of Coefficients in Singular Expansions (Stress Intensity Factors).

Let us consider the case when the maximal angle $\alpha_{M}$ is concave. Letting $\rho=\left|x-v_{M}\right|$ and $\theta$ denote the angular variable, we have from (1.10)-(1.12) that

$$
u(\rho, \theta)=k_{M} \rho^{\beta_{M}} \sin \left(\beta_{M} \theta\right)+S(x)+w(x)
$$

in a neighborhood of $v_{M}$. Here $|S(x)| \leqslant C \rho^{2 \beta_{M}}$ and if $f$ is smooth enough, $w(x)$ is a twice continuously differentiable function that vanishes on $\partial \Omega$. Since, therefore, its directional derivative at the corner vanishes in two linearly independent directions, we see that $|\omega(x)| \leqslant C \rho^{2}$. Hence,

$$
u(\rho, \theta)=k_{M} \rho^{\beta_{M}} \sin \left(\beta_{M} \theta\right)+O\left(\rho^{2 \beta_{M}}\right) \quad \text { as } \rho \rightarrow 0, \text { for } 1 / 2<\beta_{M}<1
$$

The quantity $k_{M}$ is sometimes called a stress intensity factor, and its calculation is of interest. Several ways for approximating $k_{M}$ using the information obtained in a finite element solution have been suggested, cf. the surveys by Gallagher [13] and Pian [30]. One simple method starts with the observation that with $0<\theta_{0}<\alpha_{M}$ fixed,

$$
k_{M}=\frac{u\left(\rho, \theta_{0}\right)}{\rho^{\beta_{M}} \sin \left(\beta_{M_{0}} \theta_{0}\right)}+O\left(\rho^{\beta_{M}}\right) \quad \text { as } \rho \rightarrow 0
$$

Thus,

$$
k_{M}(d, h)=\frac{u_{h}\left(d, \theta_{0}\right)}{d^{\beta_{M}} \sin \left(\beta_{M} \theta_{0}\right)}
$$

appears as a natural candidate for an approximate stress intensity factor.

The problem then presents itself as to how $d$ should be chosen. Motivated by our local analysis we offer one possibility below and give an asymptotic error estimate for this choice.

Theorem 6.1. Assume that A.1, A.2, A.3 and A.4 hold, and that $1 / 2<\beta_{M}<1$. Let $k_{M}^{h}$ be given by (6.2) with

$$
d=h^{2 / 3} \text {. }
$$


Then, given $\epsilon>0$ there exists a constant $C$ such that for $h$ sufficiently small,

$$
\left|k_{M}-k_{M}^{h}\right| \leqslant C h^{2 \beta_{M} / 3-\epsilon} .
$$

Proof. By (5.3) of Theorem 5.1 and by (6.1),

$$
\begin{aligned}
\left|k_{M}-k_{M}(d, h)\right| & \leqslant \frac{\left|u\left(d, \theta_{0}\right)-u_{h}\left(d, \theta_{0}\right)\right|}{d^{\beta_{M}} \sin \left(\beta_{M} \theta_{0}\right)}+\frac{C d^{2 \beta_{M}}}{d^{\beta_{M}}} \\
& \leqslant C\left(h^{2 \beta_{M}-\epsilon} d^{-2 \beta_{M}}+d^{\beta_{M}}\right) .
\end{aligned}
$$

This immediately gives the desired result.

A similar analysis is easily carried out for $\alpha_{M}$ convex, $\pi / 2<\alpha_{M}<\pi$. In this case the term of $O\left(\rho^{2 \beta_{M}}\right)$ in (6.1) is replaced by $O\left(\rho^{2}\right)$, and one applies a slightly varied form of Theorem 5.1. The details are left to the reader.

The information contained in the quantity $k_{M}^{h}$ can be used to improve (as far as error estimates are concerned) the approximation to $u$ near the maximal corner.

Theorem 6.2. Assume that A.1, A.2, A.3 and A.4 hold, and that $1 / 2<\beta_{M}<1$. Then with $k_{M}^{h}$ given by (6.2), (6.3), given $\epsilon>0$ there exists a constant $C$ such that

$$
\left|u(\rho, \theta)-k_{M}^{h} \rho^{\beta_{M}} \sin \left(\beta_{M} \theta\right)\right| \leqslant C h^{2 \beta_{M} / 3-\epsilon} \rho^{\beta_{M}} \text { for } \rho \leqslant h^{2 / 3} .
$$

Note that the rate of convergence in (6.5) is higher, for $\rho \leqslant h^{2 / 3}$, than that predicted by Theorem 5.1, viz. $C h^{2 \beta_{M}-\epsilon} \rho^{-\beta_{M}}, \rho \geqslant h^{1-\delta}$.

Proof. From (6.1) and (6.4) we obtain

$$
\begin{aligned}
\left|u(\rho, \theta)-k_{M}^{h} \rho^{\beta_{M}} \sin \left(\beta_{M} \theta\right)\right| & \leqslant\left|\left(k_{M}-k_{M}^{h}\right) \rho^{\beta} M \sin \left(\beta_{M} \theta\right)\right|+C \rho^{2 \beta_{M}} \\
& \leqslant C\left(h^{2 \beta_{M} / 3-\epsilon} \rho^{\beta} M+\rho^{2 \beta} M\right)
\end{aligned}
$$

which immediately implies the estimate (6.5).

Let us finally consider the approximation of higher order terms in the asymptotic expansion for $u$ around the maximal corner. For simplicity, we consider the case $3 \pi / 2<\alpha_{M}<2 \pi$. We have then, cf. (6.1)

$$
u(\rho, \theta)=k_{M} \rho^{\beta_{M}} \sin \left(\beta_{M} \theta\right)+l_{M} \rho^{2 \beta_{M}} \sin \left(2 \beta_{M} \theta\right)+O\left(\rho^{3 \beta_{M}}\right) .
$$

For $\theta_{0}$ fixed such that $\sin \left(2 \beta_{M} \theta_{0}\right) \neq 0$ and $k_{M}^{h}$ given by (6.2), (6.3), we set

$$
l_{M}(d, h)=\frac{u_{h}\left(d, \theta_{0}\right)-k_{M}^{h} d^{\beta} M \sin \left(\beta_{M} \theta_{0}\right)}{d^{2 \beta_{M}} \sin \left(2 \beta_{M} \theta_{0}\right)}
$$

as a candidate for an approximation of $l_{M}$.

Theorem 6.3. Assume that A.1, A.2, A.3 and A.4 hold, and that $1 / 2<\beta_{M}<2 / 3$. Let $l_{M}^{h}$ be given by (6.7) with

$$
d=h^{1 / 3}
$$

Then, given $\epsilon>0$ there exists a constant $C$ such that 


$$
\left|l_{M}-l_{M}^{h}\right| \leqslant C h^{\beta}{ }^{/ 3-\epsilon} .
$$

Proof. By (6.6), (6.7) we obtain

$$
\left|l_{M}-l_{M}(d, h)\right| \leqslant\left|\frac{u\left(d, \theta_{0}\right)-u_{h}\left(d, \theta_{0}\right)}{d^{2 \beta_{M}} \sin \left(2 \beta_{M_{0}} \theta_{0}\right)}\right|+\left|\frac{\left(k_{M}-k_{M}^{h}\right) d^{\beta} M}{d^{2 \beta_{M}} \sin \left(\beta_{M} \theta_{0}\right)}\right|+C d^{\left.\beta_{M} \theta_{0}\right)},
$$

and thus from (5.3) of Theorem 5.1 and from (6.4),

$$
\left|l_{M}-l_{M}(h, d)\right| \leqslant C\left(h^{2 \beta_{M}-\epsilon} d^{-3 \beta} M+h^{2 \beta} M^{13-\epsilon} d^{-\beta} M+d^{\beta} M\right) .
$$

The theorem obtains.

A similar analysis can be carried through for $3 \pi / 2>\alpha_{M}>\pi$; the order term in (6.6) is replaced by $O\left(\rho^{2}\right)$. Again we leave the details to the reader.

In a manner similar to that of Theorem 6.2, the first two terms in (6.6), with $k_{M}, l_{M}$ replaced by their approximations, can be used as an approximation for $u$ which is potentially better than $u_{h}$ is for $\rho \leqslant h^{1 / 3}$. The details are once again left to the reader.

7. Proof of Theorem 3.1. Local $H^{1}$ estimates for interior domains were proven in Nitsche and Schatz [27, Lemma 5.1] for quasi-uniform meshes. The proof of our present generalization of that result will follow closely the presentation in [27], but for completeness we shall give some of the details in the case of domains abutting on the boundary.

Liмm A.1. Assume that A.1, A.2 and A.3 hold. Let $p$ be a nonnegative integer and $D_{1} \ggg D \Omega$. There exist constants $C$ and $h_{1}>0$, depending on $p$ and $\operatorname{dist}_{\oiint}\left(D_{1}, D\right)$ such that for $0<h \leqslant h_{1}$ the following holds:

Let $u \in \overleftarrow{H}^{1}(D)$ and $u_{h} \in S^{h}(D)$ satisfy

$$
A\left(u-u_{h}, \varphi\right)=0 \text { for all } \varphi \in S^{h}(D) .
$$

Then for any $\chi \in S^{h}(D)$,

$$
\left\|u-u_{h}\right\|_{1, D} \leqslant C\left(\|u-\chi\|_{1, D}+\left\|u-u_{h}\right\|_{-p, D}\right) .
$$

In order to prove our local results here and in Section 8 we shall make use of the following auxiliary mixed boundary value problem. Let $D$ be $\Omega$ intersected with a disc, and assume that either the disc is centered at a vertex and no other vertex is contained in $D$, or that the disc is centered on $\partial \Omega$ with no vertex meeting $D$.

Let $\Gamma_{D}=\partial D \cap \partial \Omega$ and $\Gamma_{N}=\partial D \backslash \Gamma_{D}$. The problem is then to find $v$ such that with $g$ given,

$$
\begin{cases}-\Delta v=g & \text { in } D, \\ v=0 & \text { on } \Gamma_{D} \\ \partial v / \partial n=0 & \text { on } \Gamma_{N}\end{cases}
$$

In a wcak formulation we seck $v \in \overleftarrow{H}^{1}(D)$ such that 


$$
A(v, w)=(g, w) \text { for all } w \in \overleftarrow{H}^{1}(D) .
$$

Using the results of Oganesyan and Rukhovets [29] we have the following a priori estimates.

Let $D_{1} \Varangle D$ and supp $g \subseteq D_{1}$, and let $D^{\prime} \subseteq D$.

If $\bar{D}^{\prime}$ does not contain the corner or part of $\Gamma_{N}$, then for any nonnegative integer $k$ there exists a constant $C=C\left(D^{\prime}, k\right)$ such that

$$
\|v\|_{k+2, D^{\prime}} \leqslant C\|g\|_{k, D_{1}}
$$

If $\bar{D}^{\prime}$ contains no part of $\Gamma_{N}$ and if the interior angle at the corner is less than $\pi$, then there exists a constant $C=C\left(D^{\prime}\right)$ such that

$$
\|v\|_{2, D^{\prime}} \leqslant C\|g\|_{L_{2}\left(D_{1}\right)} \text {. }
$$

Proof of Lemma 7.1. By (1.5) it is enough to show the result for $D_{1}$ and $D$ the intersections with $\Omega$ of two concentric discs of radius $R$ and $2 R$, respectively, with center in $\bar{\Omega}$ and such that either

(i) the center is on $\partial \Omega$, or

(ii) the whole disc of radius $2 R$ is contained in $\Omega$.

We shall give the details in case (i) only.

We first prove that for $v_{h} \in S^{h}(D)$ such that

$$
A\left(v_{h}, \varphi\right)=0 \text { for all } \varphi \in S^{h}(D) \text {, }
$$

we have

$$
\left\|v_{h}\right\|_{1, D_{1}} \leqslant C\left\|v_{h}\right\|_{-p, D}
$$

Let $\omega \in C^{\infty}(D)$ be such that $\omega \equiv 1$ on $D_{1}$, and $\omega \equiv 0$ in a neighborhood of $\Gamma_{N}$. Then since $\bar{D}$ intersects $\partial \Omega$ where the functions in $S^{h}(D)$ vanish,

$$
\begin{aligned}
\left\|v_{h}\right\|_{1, D_{1}}^{2} & \leqslant\left\|\omega v_{h}\right\|_{1, D}^{2} \leqslant C A\left(\omega v_{h}, \omega v_{h}\right) \\
& =C A\left(v_{h}, \omega^{2} v_{h}\right)+C \int_{D} v_{h}\left\{(\Delta \omega) \omega v_{h}+2 \nabla \omega \cdot \nabla\left(\omega v_{h}\right)\right\} d x .
\end{aligned}
$$

By (7.6) and A.2 we have for a suitable $\eta \in S^{h}(D)$,

$$
A\left(v_{h}, \omega^{2} v_{h}\right)=A\left(v_{h}, \omega^{2} v_{h}-\eta\right) \leqslant C h\left\|v_{h}\right\|_{1, D}^{2}
$$

and thus,

$$
\left\|\omega v_{h}\right\|_{1, D}^{2} \leqslant C h\left\|v_{h}\right\|_{1, D}^{2}+C\left\|v_{h}\right\|_{0, D \backslash D_{1}}\left\|\omega v_{h}\right\|_{1, D}
$$

Hence it follows that

$$
\left\|v_{h}\right\|_{1, D_{1}}^{2} \leqslant C h\left\|v_{h}\right\|_{1, D}^{2}+C\left\|v_{h}\right\|_{0, D \backslash D_{1}}^{2}
$$

Next let $D_{2}$ and $D_{3}, D_{2} \Varangle D_{3}$, be the intersection of $\bar{\Omega}$ with concentric discs such that either

(i)' the center of the discs is on $\partial \Omega$, and $D_{3}$ does not contain any corner of $\Omega$, or 
(ii) $D_{3}$ is a whole disc interior to $\Omega$.

We shall only consider the case (i)' and show that for $k$ a nonnegative integer,

$$
\left\|v_{h}\right\|\left\|_{-k, D_{2}} \leqslant C h\right\| v_{h}\left\|_{1, D_{3}}+C\right\| v_{h} \|_{-k-1, D_{3}}
$$

For this, let $\omega \in \mathcal{C}^{\infty}\left(D_{3}\right)$ with $\omega \equiv 1$ on $D_{2}$, and $\omega \equiv 0$ near $\partial D_{3} \backslash\left(\partial D_{3} \cap \partial \Omega\right)$. We have

$$
\left\|v_{h}\right\|_{-k, D_{2}}=\sup _{\varphi \in C^{\infty}\left(D_{2}\right)} \frac{\left(\omega v_{h}, \varphi\right)}{\|\varphi\|_{k, D_{2}}} .
$$

For fixed $\varphi$, let $\psi$ solve the auxiliary mixed problem (7.3) over $D$, assuming after a change of $D$ if necessary that $D_{3} D$. Note that by (7.4),

$$
\|\psi\|_{k+2, D_{3}} \leqslant C\|\varphi\|_{k, D_{2}}
$$

We have now

$$
\left(\omega v_{h}, \varphi\right)=A\left(\omega v_{h}, \psi\right)=A\left(v_{h}, \omega \psi\right)+\int v_{h}((\Delta \omega) \psi+2 \nabla \omega \cdot \nabla \psi) d x
$$

Writing $A\left(v_{h}, \omega \psi\right)=A\left(v_{h}, \omega \psi-\chi\right)$ for a suitable $\chi$ we get upon using (2.7) of Lemma 2.1 and (7.10),

$$
\begin{aligned}
\left(\omega v_{h}, \varphi\right) & \leqslant C\left\|v_{h}\right\|_{1, D_{3}} h\|\psi\|_{2, D_{3}}+\left\|v_{h}\right\|_{-k-1, D_{3}}\|\psi\|_{k+2, D_{3}} \\
& \leqslant C\left(h\left\|v_{h}\right\|_{1, D_{3}}+\left\|v_{h}\right\| \|_{-k-1, D_{3}}\right)\|\varphi\|_{k, D_{2}} .
\end{aligned}
$$

Thus (7.9) obtains.

Iterating (7.8) and (7.9) over suitable domains inserted between the original $D_{1}$ and $D$, we obtain with $D_{1} \$ D^{\prime} \$$,

$$
\left\|v_{h}\right\|_{1, D_{1}} \leqslant C h^{\gamma(p+1)}\left\|v_{h}\right\|_{1, D^{\prime}}+C\left\|v_{h}\right\|_{-p, D^{\prime}}
$$

In view of A.3, the result (7.7) follows.

We can now show (7.2). Let $D_{1}$ and $D$ be as in case (i) and $D_{1} \$ D^{\prime} \$ D$. Let $\omega \in C^{\infty}(D)$ be such that $\omega \equiv 1$ on $D^{\prime}, \omega \equiv 0$ in a neighborhood of $\partial D \backslash(\partial D \cap \partial \Omega)$. Let $\tilde{u}=\omega u$ and let $P \tilde{u} \in S^{h}(D)$ be the solution of

$$
A(\tilde{u}-\tilde{u}, \varphi)=0 \text { for all } \varphi \in S^{h}(D) .
$$

Clearly then,

$$
\|\tilde{u}-P \tilde{u}\|_{1, D} \leqslant C\|\tilde{u}\|_{1, D}
$$

Next note that $A\left(P \tilde{u}-u_{h}, \varphi\right)=0$ for $\varphi \in S^{h}\left(D^{\prime}\right)$ so that by (7.7),

$$
\begin{aligned}
\left\|P \tilde{u}-u_{h}\right\|_{1, D_{1}} & \leqslant C\left\|P \tilde{u}-u_{h}\right\|_{-p, D^{\prime}} \leqslant C\|\tilde{u}-P \tilde{u}\|_{1, D}+C\left\|\tilde{u}-u_{h}\right\|_{-p, D^{\prime}} \\
& \leqslant C\|u\|_{1, D}+C\left\|u-u_{h}\right\|_{-p, D} .
\end{aligned}
$$

Thus,

$$
\left\|u-u_{h}\right\|_{1, D_{1}} \leqslant C\|u\|_{1, D}+C\left\|u-u_{h}\right\|_{-p, D}
$$


and (7.2) follows upon writing $u-u_{h}=(u-\chi)+\left(\chi-u_{h}\right)$.

This completes the proof of the lemma.

As in [31, Lemma 3.2] we have the following result in the case when the domains $D_{1}$ and $D$ are allowed to vary and Become small with $h$.

LemmA 7.2. Assume that A.1, A.2, A.3 and A.4 hold. Let $p$ be a nonnegative integer and $0<\delta \leqslant 1$. There exist constants $C=C(p, \delta)$ and $h_{2}=h_{2}(p, \delta)>0$ such that the following holds.

Let $D_{1} \$ with $\operatorname{dist}_{\oiint}\left(D_{1}, D\right)=d \geqslant h^{1-\delta}$, and let $u \in \overleftarrow{H}^{1}(D)$ and $u_{h} \in S^{h}(D)$ be such that

$$
A\left(u-u_{h}, \varphi\right)=0 \text { for all } \varphi \in S^{h}(D) .
$$

Then for $h \leqslant h_{2}$ we have for any $\chi \in S^{h}(D)$,

$$
\begin{aligned}
& d^{-1}\left\|u-u_{h}\right\|_{0, D_{1}}+\left|u-u_{h}\right|_{1, D_{1}} \\
& \quad \leqslant C\left(d^{-1}\|u-\chi\|_{0, D}+|u-\chi|_{1, D}+d^{-1-p}\left\|u-u_{h}\right\|_{-p, D}\right)
\end{aligned}
$$

Since $h / d \leqslant h^{\delta} \leqslant h_{1}$ for $h$ sufficiently small the lemma follows from Lemma 7.1 by a scaling argument using the hypothesis A.4; see [31, Lemma 3.2] for details.

We can now prove Theorem 3.1.

Proof of Theorem 3.1. Let $\left\|u-u_{h}\right\|_{L_{\infty}\left(D_{1}\right)}=\left|\left(u-u_{h}\right)\left(x_{0}\right)\right|$ for $x_{0} \in \bar{D}_{1}$. Let $D_{2}$ and $D_{3}$ be the intersections of $D$ with discs of radius $d / 4$ and $d / 2$ centered at $x_{0}$. Then for any $\chi \in S^{h}(D)$,

$$
\left|\left(u-u_{h}\right)\left(x_{0}\right)\right| \leqslant\|u-\chi\|_{L_{\infty}\left(D_{2}\right)}+\left\|\chi-u_{h}\right\|_{L_{\infty}\left(D_{2}\right)} .
$$

Using A.3 we have for $q$ such that $\gamma 2 / q=\epsilon$,

$$
\left\|\chi-u_{h}\right\|_{L_{\infty}\left(D_{2}\right)} \leqslant C h^{-\epsilon}\left\|\chi-u_{h}\right\|_{L_{q}\left(D_{3}\right)}
$$

Applying Sobolev's inequality to $D_{3}$ we obtain

$$
\begin{aligned}
\left\|\chi-u_{h}\right\|_{L_{q}\left(D_{3}\right)} \leqslant C d^{2 / q}\left(\left|\chi-u_{h}\right|_{1, D_{3}}+d^{-1}\left\|\chi-u_{h}\right\|_{0, D_{3}}\right) & \\
\leqslant C\left(|u-\chi|_{1, D_{3}}+d^{-1}\|u-\chi\|_{0, D_{3}}\right. & +\left|u-u_{h}\right|_{1, D_{3}} \\
& \left.+d^{-1}\left\|u-u_{h}\right\|_{0, D_{3}}\right) .
\end{aligned}
$$

An application of Lemma 7.2 completes the proof.

8. Proof of Theorem 3.2. We shall prove the following generalization of the estimates of [31].

Lemma 8.1. Assume that A.1, A.2, A.3 and A.4 hold. Let $p$ be a nonnegative integer, and $\epsilon>0$. Furthermore let $D_{1} \Varangle D$ where $D$ does not contain any concave corner of $\Omega$. There exist constants $C$ and $h_{3}>0$ depending on $p, \epsilon$, and $\operatorname{dist}_{\Varangle}\left(D_{1}, D\right)$ such that for $h \leqslant h_{3}$ the following holds.

Let $u \in \stackrel{H}{H}^{1}(D) \cap W_{\infty}^{1}(D)$ and $u_{h} \in S^{h}(D)$ satisfy 


$$
A\left(u-u_{h}, \varphi\right)=0 \text { for all } \varphi \in S^{h}(D) \text {. }
$$

Then for any $\chi \in S^{h}(D)$,

(8.2) $\left\|u-u_{h}\right\|_{L_{\infty}\left(D_{1}\right)} \leqslant C h^{-\epsilon}\left(h|u-\chi|_{w_{\infty}^{1}(D)}+\|u-\chi\|_{L_{\infty}(D)}+\left\|u-u_{h}\right\|_{-p, D}\right)$.

Theorem 3.2 follows from Lemma 8.1 by use of a scaling argument, cf. [31] for details.

In the proof of Lemma 8.1 we shall need two simple auxiliary results. The first concerns the mixed problem (7.3) when the right-hand side has small support.

Lemma 8.2. For any $\epsilon>0$ there exists a constant $C=C(D, \epsilon)$ such that the following holds. Let $g$ be supported in a domain $D_{1} \subseteq D$, with diam $D_{1}=d$. Then

$$
\|v\|_{1, D} \leqslant C d^{1-\epsilon}\|g\|_{L_{2}\left(D_{1}\right)}
$$

Proof. We have

$$
\|v\|_{1, D}^{2} \leqslant C A(v, v)=C(g, v),
$$

and using Hölder's inequality with $1 / q+1 / q^{\prime}=1$ and $q$ large,

$$
\begin{aligned}
|(g, v)| & \leqslant C\|g\|_{L_{q^{\prime}}\left(D_{1}\right)}\|v\|_{L_{q}(D)} \\
& \leqslant C d^{\left(2-q^{\prime}\right) / q^{\prime}}\|g\|_{L_{2}\left(D_{1}\right)}\|v\|_{L_{q}(D)} .
\end{aligned}
$$

By Sobolev's inequality, for $q<\infty$,

$$
\|v\|_{L_{q}(D)} \leqslant C(q, D)\|v\|_{1, D}
$$

and the result follows since $q^{\prime}$ can be taken arbitrarily close to 1 .

We shall also require the following result concerning harmonic functions.

Lemma 8.3. Let $D_{1} \ggg D_{2}$ with dist $\left(D_{1}, D_{2}\right)=d$, diam $D_{1} \leqslant d$, diam $D_{2} \leqslant$ $3 d$, and such that $D_{2}$ contains no concave corner of $\Omega$. There exists a constant $C$ such that the following holds:

For $v$ harmonic on $D_{2}$ and vanishing on $\partial D_{2} \cap \partial \Omega$,

$$
\|v\|_{2, D_{1}} \leqslant C d^{-1}\|v\|_{1, D_{2}} \text {. }
$$

Proof. By a covering argument it suffices to prove (8.4) in two cases.

(i) $D_{2}$ and $D_{1}$ are concentric discs of radius $2 d$ and $d$, respectively with $D_{2}$ interior to $\Omega$.

(ii) $D_{2}$ and $D_{1}$ are the intersections with $\Omega$ of two concentric discs of radius $2 d$ and $d$, respectively, and with center on $\partial \Omega$ such that either (a) $D_{2}$ contains no vertex of $\Omega$, or (b) the center is at a vertex of convex interior angle.

In case (i) we note that $w=\partial v / \partial x_{i}$ is also harmonic. Thus let us show that for a harmonic function $w$,

$$
\|\nabla w\|_{0, D_{1}} \leqslant C d^{-1}\|w\|_{0, D_{2}} .
$$

Let $\omega$ be a $C^{\infty}$ cut-off function such that $\omega \equiv 1$ on $D_{1}$, supp $\omega \subset \subset D_{2}$, and 


$$
\left\|D^{\alpha} \omega\right\|_{L_{\infty}} \leqslant C d^{-|\alpha|}
$$

We have $\|\nabla w\|_{0, D_{1}} \leqslant\|\omega \nabla w\|_{0}^{2}$, and

$$
\|\omega \nabla w\|_{0}^{2}=\int(\omega \nabla w) \cdot(\omega \nabla w)=\int \nabla w \cdot\left(\nabla\left(\omega^{2} w\right)\right)-2 \int \omega \nabla w \cdot(\nabla \omega) w .
$$

The first term on the right is zero, so that

$$
\|\omega \nabla w\|_{0}^{2} \leqslant 2\|\omega \nabla w\|_{0} C d^{-1}\|w\|_{0, D_{2}} .
$$

Thus,

$$
\|\omega \nabla w\|_{0} \leqslant C d^{-1}\|w\|_{0, D_{2}}
$$

and (8.5) follows.

For case (ii), let us treat the situation when the discs are centered at a convex angle. Letting $\omega$ be a $C^{\infty}$ cut-off function which is $\equiv 1$ on $D_{1}$, vanishes in a neighborhood of $\partial D_{2} \backslash\left(\partial D_{2} \cap \partial \Omega\right)$, and satisfies (8.6), we have upon using the a priori estimate (7.5),

$$
\begin{aligned}
\|v\|_{2, D_{1}} & \leqslant C\|\Delta(\omega v)\|_{0, D_{2}} \\
& \leqslant C\left(d^{-1}\|v\|_{1, D_{2} \backslash D_{1}}+d^{-2}\|v\|_{0, D_{2} \backslash D_{1}}\right) .
\end{aligned}
$$

Since $\operatorname{diam}\left(D_{2} \backslash D_{1}\right) \leqslant d$ and $v$ vanishes on $\partial\left(D_{2} \backslash D_{1}\right) \cap \partial \Omega$, we have by Poincaré's inequality that

$$
\|v\|_{0, D_{2} \backslash D_{1}} \leqslant C d\|\nabla v\|_{0, D_{2} \backslash D_{1}}
$$

The desired inequality (8.4) obtains.

This completes the proof of Lemma 8.3.

We can now prove Lemma 8.1.

Proof. We shall assume that $D_{1}$ and $D$ are the intersections of $\bar{\Omega}$ with concentric discs such that either

(i) (a) the discs are centered at a convex corner of $\Omega$ and contain no other corner, or

(i) (b) the discs are centered on a straight line segment of $\partial \Omega$ and contain no corner, or

(ii) the discs are interior to $\Omega$.

We shall give the details of the proof in the case (i) (a). This reasoning immediately carries over to the case (i) (b). In the situation of (ii), one first perturbs the bilinear form $A$ into $\widetilde{A}(v, w)=A(v, w)+(v, w)$ which is then coercive over $H^{1}$. The procedure in (i) applies if $A$ is replaced by $\widetilde{A}$ in (8.1). To conclude the argument one then proceeds as in [31, Appendix 1].

We shall first reduce the proof to finding estimates for a locally defined problem. Let $D_{1} D_{2} \Varangle D_{3} \oiint D$ be intersections of $\Omega$ with discs centered at the convex corner. Let $\omega \in C^{\infty}(D)$ with $\omega \equiv 1$ on $D_{2}, \omega \equiv 0$ outside $D_{3}$. Set $\tilde{u}=\omega u$ and let $\tilde{u}_{h} \in S^{h}(D)$ be the solution of 


$$
A\left(\tilde{u}-\tilde{u}_{h}, \varphi\right)=0 \text { for all } \varphi \in S^{h}(D) .
$$

Note that since $\bar{D}$ intersects $\partial \Omega$, there is a unique $\tilde{u}_{h}$ satisfying (8.7).

We shall derive the following estimate for $\tilde{u}-\widetilde{u}_{h}$.

LEMMA 8.4. Under the conditions of Lemma 8.1,

$$
\left\|\tilde{u}-\tilde{u}_{h}\right\|_{L_{\infty}\left(D_{2}\right)} \leqslant C h^{-\epsilon}\left(h\|\tilde{u}\|_{W_{\infty}^{1}(D)}+\|\tilde{u}\|_{L_{\infty}(D)}\right) .
$$

Assuming the validity of Lemma 8.4 for the moment, let us complete the proof of Lemma 8.1. We have

$$
\left\|u-u_{h}\right\|_{L_{\infty}\left(D_{1}\right)} \leqslant\left\|\tilde{u}-\tilde{u}_{h}\right\|_{L_{\infty}\left(D_{1}\right)}+\left\|\tilde{u}_{h}-u_{h}\right\|_{L_{\infty}\left(D_{1}\right)} .
$$

Note now that $A\left(\tilde{u}_{h}-u_{h}, \varphi\right)=0$ for $\varphi$ in $S^{h}\left(D_{2}\right)$. Let $D_{1} \Varangle D_{1}^{\prime} \ D_{2}$. Using A.3, Sobolev's inequality (1.6), (7.2) with $u=0$, and Lemma 8.4 with $\epsilon$ replaced by $\epsilon / 2$, we obtain for $q=4 \gamma / \epsilon$,

$$
\begin{aligned}
\left\|\tilde{u}_{h}-u_{h}\right\|_{L_{\infty}\left(D_{1}\right)} & \leqslant C h^{-\epsilon / 2}\left\|\tilde{u}_{h}-u_{h}\right\|_{L_{q}\left(D_{1}^{\prime}\right)} \\
& \leqslant C h^{-\epsilon / 2}\left\|\tilde{u}_{h}-u_{h}\right\|_{1, D_{1}^{\prime}} \leqslant C h^{-\epsilon / 2}\left\|\tilde{u}_{h}-u_{h}\right\|_{-p, D_{2}} \\
& \leqslant C h^{-\epsilon / 2}\left(\left\|\tilde{u}-\tilde{u}_{h}\right\|_{L_{\infty}\left(D_{2}\right)}+\left\|u-u_{h}\right\| \|_{-p, D}\right) \\
& \leqslant C h^{-\epsilon}\left(h|\tilde{u}|_{w_{\infty}^{1}(D)}+\|\tilde{u}\|_{L_{\infty}(D)}\right)+C h^{-\epsilon / 2}\left\|u-u_{h}\right\|_{-p, D} .
\end{aligned}
$$

Using this and Lemma 8.4 again in (8.9), we arrive at

$$
\left\|u-u_{h}\right\|_{L_{\infty}\left(D_{1}\right)} \leqslant C h^{-\epsilon}\left(h|u|_{W_{\infty}(D)}^{1}+\|u\|_{L_{\infty}(D)}+\left\|u-u_{h}\right\|_{-p, D}\right) .
$$

The inequality (8.2) follows upon writing $u-u_{h}=(u-\chi)+\left(\chi-u_{h}\right)$ for any $\chi \in$ $S^{h}(D)$.

It remains to prove Lemma 8.4.

Clearly, it suffices to show that

$$
\left\|\tilde{u}_{h}\right\|_{L_{\infty}\left(D_{2}\right)} \leqslant C h^{-\epsilon}\left(h|\tilde{u}|_{W_{\infty}^{1}(D)}+\|\tilde{u}\|_{L_{\infty}(D)}\right) .
$$

Let $\left\|\tilde{u}_{h}\right\|_{L_{\infty}\left(D_{2}\right)}=\left|u_{h}\left(x_{0}\right)\right|$ for $x_{0} \in \bar{D}_{2}$, and let $\Lambda_{1}$ and $\Lambda_{2}$ be the intersections with $D$ of discs of radius $\rho=h^{1-\delta}$ and $2 \rho$, respectively, centered at $x_{0}$. Here $\delta=\epsilon / 3$. By A.3 and Sobolev's inequality we obtain for $q=2 \gamma / \delta$,

$$
\left|\tilde{u}_{h}\left(x_{0}\right)\right| \leqslant C h^{-\delta}\left\|\tilde{u}_{h}\right\|_{L_{q}\left(\Lambda_{1}\right)} \leqslant C h^{-\delta}\left(\left\|\tilde{u}_{h}\right\|_{1, \Lambda_{1}}+\frac{1}{\rho}\left\|\tilde{u}_{h}\right\|_{0, \Lambda_{1}}\right) .
$$

Thus,

$$
\left|\tilde{u}_{h}\left(x_{0}\right)\right| \leqslant C h^{-\delta}\|\tilde{u}\|_{1, \Lambda_{1}}+C h^{-\delta}\left\|\tilde{u}-\tilde{u}_{h}\right\|_{1, \Lambda_{1}}+\frac{C h^{-\delta}}{\rho}\left\|\tilde{u}_{h}\right\|_{0, \Lambda_{1}} .
$$

Now,

$$
\|\widetilde{u}\|_{1, \Lambda_{1}} \leqslant C \rho\|\tilde{u}\|_{W_{\infty}^{1}(D)}=C h^{1-\delta}\|\tilde{u}\|_{W_{\infty}^{1}(D)} .
$$


From Lemma 7.2 with $p=0$ and $\chi \equiv 0$ we have

$$
\left\|\tilde{u}-\tilde{u}_{h}\right\|_{1, \Lambda_{1}} \leqslant C\left(|\tilde{u}|_{1, \Lambda_{2}}+\frac{1}{\rho}\|\tilde{u}\|_{0, \Lambda_{2}}+\frac{1}{\rho}\left\|\tilde{u}_{h}\right\|_{0, \Lambda_{2}}\right)
$$

and using this and (8.12) in (8.11),

$$
\begin{aligned}
\left|\tilde{u}_{h}\left(x_{0}\right)\right| & \leqslant C h^{1-2 \delta}\|\tilde{u}\|_{w_{\infty}^{1}(D)}+C h^{-\delta}|\tilde{u}|_{1, \Lambda_{2}}+\frac{C h^{-\delta}}{\rho}\|\tilde{u}\|_{0, \Lambda_{2}}+\frac{C h^{-\delta}}{\rho}\left\|\tilde{u}_{h}\right\|_{0, \Lambda_{2}} \\
& \leqslant C h^{1-2 \delta}\|\tilde{u}\|_{w_{\infty}^{1}(D)}+C h^{-\delta}\|\tilde{u}\|_{L_{\infty}\left(\Lambda_{2}\right)}+C h^{-1}\left\|\tilde{u}_{h}\right\|_{0, \Lambda_{2}} .
\end{aligned}
$$

The problem of showing (8.10), and hence Lemma 8.4 , is now reduced to verifying that

$$
h^{-1}\left\|\tilde{u}_{h}\right\|_{0, \Lambda_{2}} \leqslant C h^{-\epsilon}\left(h\|\tilde{u}\|_{w_{\infty}^{1}(D)}+\|\tilde{u}\|_{L_{\infty}(D)}\right)
$$

We have

$$
\left\|\tilde{u}_{h}\right\|_{0, \Lambda_{2}}=\sup _{\varphi \in C_{0}^{\infty}\left(\Lambda_{2}\right)} \frac{\left(\tilde{u}_{h}, \varphi\right)}{\|\varphi\|_{0, \Lambda_{2}}} .
$$

For each fixed $\varphi$, let $v \in \stackrel{\zeta}{H}^{1}(D)$ solve the problem

$$
A(\psi, v)=(\psi, \varphi) \text { for all } \psi \in \stackrel{\zeta}{H}^{1}(D) .
$$

Letting $v_{h} \in S^{h}(D)$ be such that $A\left(\chi, v-v_{h}\right)=0$ for all $\chi \in S^{h}(D)$ we obtain then

$$
\left(\tilde{u}_{h}, \varphi\right)=A\left(\tilde{u}_{h}, v\right)=A\left(\tilde{u}_{h}, v_{h}\right)=A\left(\tilde{u}, v_{h}\right)=A\left(\tilde{u}, v_{h}-v\right)+(\tilde{u}, \varphi)
$$

Note next that

$$
|(\tilde{u}, \varphi)| \leqslant C \rho\|\tilde{u}\|_{L_{\infty}(D)}\|\varphi\|_{0, \Lambda_{2}}=C h^{1-\delta}\|\tilde{u}\|_{L_{\infty}(D)}\|\varphi\|_{0, \Lambda_{2}} .
$$

Also,

$$
\left|A\left(\tilde{u}, v-v_{h}\right)\right| \leqslant C\|\tilde{u}\|_{w_{\infty}^{1}\left(D_{2}\right)}\left\|v-v_{h}\right\|_{w_{1}^{1}\left(D_{2}\right)}
$$

Using (8.14) through (8.18) we see that (8.13) would follow if

$$
\left\|v-v_{h}\right\|_{w_{1}^{1}\left(D_{2}\right)} \leqslant C h^{2-\epsilon}\|\varphi\|_{0, \Lambda_{2}}
$$

For simplicity in notation, let the radii of the discs whose intersection with $\Omega$ form $D_{2}$ and $D$ be $1 / 8$ and 1 , respectively, and set

$$
\begin{aligned}
& \Omega_{j}=\left\{x: 2^{-j-1} \leqslant\left|x-x_{0}\right| \leqslant 2^{-j}\right\} \cap D, \quad j=-1, \ldots, J+2, \\
& \Omega_{j}^{l}=\left(\Omega_{j-l} \cup \bar{\Omega}_{j-l+1} \cup \cdots \cup \Omega_{j+l}\right), \quad l=1,2,3,
\end{aligned}
$$

where

$$
J=\left[\ln _{2} \frac{1}{16 h^{1-\delta}}\right]+1 \quad([\alpha] \text { denotes the integral part of } \alpha)
$$


Let also

$$
\Omega_{I}=\left\{x:\left|x-x_{0}\right| \leqslant 2^{-J}\right\} \cap D .
$$

Then

$$
\left\|v-v_{h}\right\|_{w_{1}^{1}\left(D_{2}\right)}=\left\|v-v_{h}\right\|_{w_{1}^{1}\left(\Omega_{I}\right)}+\sum_{j=3}^{J}\left\|v-v_{h}\right\|_{w_{1}^{1}\left(\Omega_{j}\right)}
$$

$$
\leqslant C\left(h^{1-\delta}\left\|v-v_{h}\right\|_{1, \Omega_{I}}+\sum_{j=3}^{J} 2^{-j}\left\|v-v_{h}\right\|_{1, \Omega_{j}}\right)
$$

We first consider the domain $\Omega_{I}$. Here we use (8.3) of Lemma 8.2 which says that

$$
\|v\|_{1, D} \leqslant C \rho^{1-\delta}\|\varphi\|_{0, \Lambda_{2}} \leqslant C h^{1-2 \delta}\|\varphi\|_{0, \Lambda_{2}}
$$

Thus,

$$
\left\|v-v_{h}\right\|_{1, D} \leqslant C\|v\|_{1, D} \leqslant C h^{1-2 \delta}\|\varphi\|_{0, \Lambda_{2}}
$$

Next apply (7.12) of Lemma 7.2 to the domains $\Omega_{j} \ggg \Omega_{j}^{1}$. It follows that

$$
I \equiv \sum_{j=3}^{J} 2^{-j}\left\|v-v_{h}\right\|_{1, \Omega_{j}} \leqslant \sum_{j=3}^{J}\left(2^{-j}\|v-\chi\|_{1, \Omega_{j}^{1}}+\|v-\chi\|_{0, \Omega_{j}^{1}}+\left\|v-v_{h}\right\|_{0, \Omega_{j}^{1}}\right) .
$$

Using the approximation result (2.7) of Lemma 2.1, we obtain

$$
I \leqslant C \sum_{j=3}^{J} 2^{-j} h\|v\|_{2, \Omega_{j}^{2}}+C \ln \frac{1}{h}\left\|v-v_{h}\right\|_{0, D^{\prime}}
$$

where $D^{\prime}$ is the intersection with $\Omega$ of a disc of radius $1 / 4$. Since $v$ is harmonic outside of $\Lambda_{2}$, we have from (8.4), as $D$ contains no concave corner, that

$$
\|v\|_{2, \Omega_{j}^{2}} \leqslant C 2^{j}\|v\|_{1, \Omega_{j}^{3}} \text {. }
$$

A simple duality argument employing the a priori estimate (7.5), and (2.7) of Lemma 2.1 establishes that

$$
\left\|v-v_{h}\right\|_{0, D^{\prime}} \leqslant C h\|v\|_{1, D^{\prime}}
$$

Using this and (8.23) in (8.22), we find

$$
I \leqslant C h \ln \frac{1}{h}\|v\|_{1, D}
$$

By another application of (8.3) we obtain that for $\ln (1 / h) \leqslant h^{-\delta}$,

$$
I \leqslant C h^{2-2 \delta}\|\varphi\|_{0, \Lambda_{2}}
$$

Inserting this and (8.21) into (8.20), we have proven that

$$
\left\|v-v_{h}\right\|_{w_{1}^{1}\left(D_{2}\right)} \leqslant C h^{2-3 \delta}\|\varphi\|_{0, \Lambda_{2}} \leqslant C h^{2-\epsilon}\|\varphi\|_{0, \Lambda_{2}} .
$$

This is the desired estimate (8.19), which completes the proof of Lemma 8.4.

The verification of Lemma 8.1 is now complete. 
9. Examples of Finite Element Spaces. We shall give examples of finite element spaces that satisfy our assumptions A.1-A.4.

The spaces described in our first example are well known and we shall be brief.

Example 1. Quasi-Uniform Meshes. Consider a triangular or quadrilateral quasiuniform partition of $\Omega$. As function spaces $S^{h}(\Omega)$ we may use the Lagrange or Hermite elements, see Ciarlet and Raviart [8], the elements of Bramble and Zlámal [6], the tensor products of one dimensional piecewise polynomials, and others.

For the well-known techniques involved in verifying our assumptions, cf. [8] or Ciarlet [7]. In connection with A.2 (where now $\gamma=1$ ), see also Nitsche and Schatz [27].

Our next example is a simple one on a non-quasi-uniform mesh.

Example 2. Piecewise Bilinear Functions on a Non-Quasi-Uniform Mesh. Let $\partial \Omega$ consist of axis parallel line segments, and consider a sequence of partitions of $\Omega$ into axis parallel rectangles $\tau_{h, i}, i=1, \ldots, I(h)$. Let $l_{1, h, i}$ and $l_{2, h, i}$ denote the lengths of the sides of $\tau_{h, i}$, and assume that with $K_{1}, K_{2}>0$ and $\gamma_{1} \geqslant 1$ constants,

$$
\left\{\begin{array}{l}
\max _{i}\left(\max _{j=1,2}\left(l_{j, h, i}\right)\right) \leqslant K_{1} h, \\
\min _{i}\left(\min _{j=1,2}\left(l_{j, h, i}\right)\right) \geqslant K_{2} h^{\gamma_{1}} .
\end{array}\right.
$$

Let $S^{h}(\Omega)$ consist of the continuous functions on $\Omega$ which vanish on $\partial \Omega$, and which on each rectangle $\tau_{h, i}$ reduce to a bilinear function $a_{0}+a_{1} x+a_{2} y+a_{3} x y$.

If $T$ denotes any rectangle of sidelengths $l_{1}, l_{2}$ with $L=\max \left(l_{1}, l_{2}\right)$ bounded, $\leqslant 1$ say, then Jamet [16] has shown that with $\chi$ equal to the interpolant of $v$ at the corners,

$$
\|v-\chi\|_{L_{\infty}(T)}+L\|u-\chi\|_{w_{\infty}^{1}(T)} \leqslant C L^{2}|v|_{W_{\infty}^{2}(T)}
$$

where $C$ is independent of $l_{1}$ or $l_{2}$. From this we obtain A.1 with $r=2$ and the part of A.4 that pertains to A.1.

In Appendix 1 we shall show that for $\chi$ a bilinear function on $T, \omega$ in $C^{3}(T)$ and with $\eta$ denoting the interpolant of $\omega \chi$,

$$
\|\omega \chi-\eta\|_{1, T} \leqslant C L\|\omega\|_{W_{\infty}^{3}(T)}\|\chi\|_{1, T} .
$$

From this we deduce that A. 2 and the corresponding part of A.4 are true, with $l_{0}=3$.

To verify A.3 (we consider here only the second part) we show in Appendix 1 that with $l=\min \left(l_{1}, l_{2}\right)$,

$$
\|\chi\|_{1, T} \leqslant C l^{-(p+1)} \mid\|\chi\|_{-p, T}
$$

where again $C$ is independent of $l_{1}$ and $l_{2}$. Using (9.1) and (1.5), the inequality (2.4) of A.3 follows with $\gamma=\gamma_{1}$. For the corresponding part of A.4, note that it follows from (9.1) that on a rectangle that is transformed via a stretching by a factor $1 / d$,

$$
l \geqslant K_{2} \frac{h^{\gamma_{1}}}{d}
$$


Since for $d \geqslant h^{1-\delta}$,

$$
\frac{h^{\gamma_{1}}}{d} \geqslant\left(\frac{h}{d}\right)^{1+\left(\gamma_{1}-1\right) / \delta}
$$

we see that A.4 holds.

Example 3. Piecewise Linear Functions on a Non-Quasi-Uniform Triangulation. Assume that $\Omega$ is partitioned into triangles $\tau_{h, i}, i=1, \ldots, I(h)$. Let $\theta_{h, i}$ be the maximum interior angle of $\tau_{h, i}$, and assume that with $\theta_{0}<\pi$ independent of $h$,

$$
\max _{i} \theta_{h, i} \leqslant \theta_{0}
$$

Furthermore, let $\rho_{h, i}=\operatorname{diam} \tau_{h, i}$, and let $\sigma_{h, i}=\max$ \{diameter of discs inscribed in $\left.\tau_{h, i}\right\}$. Assume that with $K_{1}, K_{2}>0$ and $\gamma_{1} \geqslant 1$ constants,

$$
\left\{\begin{array}{l}
\max _{i} \rho_{h, i} \leqslant K_{1} h, \\
\min _{i} \sigma_{h, i} \geqslant K_{2} h^{\gamma_{1}} .
\end{array}\right.
$$

Let $S^{h}(\Omega)$ consist of the continuous functions on $\Omega$ which vanish on $\partial \Omega$, and on each triangle $\tau_{h, i}$ reduce to a linear function $a_{0}+a_{1} x+a_{2} y$.

To verify our assumptions in this case, let $T$ be an arbitrary triangle, and let $\theta \leqslant \theta_{0}, \rho, \sigma \leqslant 1$ have the obvious meanings. For $\chi$ the interpolant of $v$ we have, Jamet [16],

$$
\|v-\chi\|_{L_{\infty}(T)}+\rho\|v-\chi\|_{W_{\infty}^{1}(T)} \leqslant C\left(\theta_{0}\right) \rho^{2}|v|_{W_{\infty}^{2}(T)} .
$$

Thus A.1 with $r=2$ and the corresponding part of A.4 follow.

To verify A.2 we quote the following result from Babuska-Aziz [3]. For $v \in$ $H^{2}(T)$ and $\eta$ the interpolant,

$$
\|v-\eta\|_{1, T} \leqslant C\left(\theta_{0}\right) \rho\|v\|_{2, T}
$$

Letting $\chi$ be a linear function and $\eta$ the interpolant of $\omega \chi$ where $\omega \in C^{2}(T)$, we obtain from (9.5) since second derivatives of a linear function vanish,

$$
\|\omega \chi-\eta\|_{1, T} \leqslant C\left(\theta_{0}\right) \rho\|\omega\|_{w_{\infty}^{2}(T)}\|\chi\|_{1, T}
$$

This proves A.2 and the corresponding part of A.4.

The inverse assumption A.3 is taken care of in a manner similar to that of Example 2, using (9.4).

In Part 2 of this series we shall give a more detailed analysis of the finite element method when the mesh is refined near a vertex. The results obtained in Theorems 3.1 and 3.2 are valid for a large class of meshes which are associated with refinements, and will be of importance in the derivation of results in Part 2. As our last example we shall describe certain mesh refinements which satisfy our hypotheses A.1-A.4.

Example 4. A Special Class of Locally Quasi-Uniform Meshes. Let $\Omega_{j}, j=1$, $\ldots, M$, be the intersection of $\Omega$ with a disc of radius $R_{j}$ centered at the $j$ th corner vertex $v_{j}$, and such that $\Omega_{j}$ contains no other corner. Set $\Omega_{0}=\Omega \backslash\left(\bigcup_{j=1}^{M} \bar{\Omega}_{j}\right)$. 
Assume that the partition is quasi-uniform over $\Omega_{0}$ with the diameter of any element being equivalent to $h$. Let

$$
\begin{aligned}
\Omega_{j, k}=\left\{x: 2^{-k} R_{j} \leqslant\left|x-v_{j}\right| \leqslant 2^{-k+1} R_{j}\right\} & \cap \Omega, \\
& j=1, \ldots, M, k=1, \ldots, k_{j},
\end{aligned}
$$

and

$$
\Omega_{j, k_{j}+1}=\left\{x:\left|x-v_{j}\right| \leqslant 2^{\left.-k_{j} R_{j}\right\} \cap \Omega .}\right.
$$

On each $\Omega_{j, k}$ let the partition involved be quasi-uniform with the diameter of the elements involved being comparable to $h_{j, k}$, where with $\gamma_{1} \geqslant 1$ constant,

$$
h^{\gamma_{1}} \leqslant h_{j, k} \leqslant h, \quad j=1, \ldots, M, k=1, \ldots, k_{j}+1 .
$$

Consider on these kinds of meshes, e.g. the finite element spaces listed in Example 1. One may verify the assumptions A.1-A.4 essentially as in the quasi-uniform case. In A.3, $\gamma$ may be taken as $\gamma_{1}$.

Appendix 1. Verification of Assumptions A.2 and A.3 in Example 2 of Section 9. Let $T$ be a rectangle with corners $P_{0}=(0,0), P_{1}=\left(l_{1}, 0\right), P_{2}=\left(l_{1}, l_{2}\right)$ and $P_{3}=\left(0, l_{2}\right)$. Here it is only assumed that $l_{1}$ and $l_{2}$ are bounded, say by unity. Put $L=\max \left(l_{1}, l_{2}\right)$ and $l=\min \left(l_{1}, l_{2}\right)$.

We shall first verify the assumption A.2 and its counterpart in A.4. To accomplish this, we need to investigate in some detail the interpolant into $S^{h}$ of a function $v$. Given $v$, let $v_{i}=v\left(P_{i}\right), i=0,1,2,3$. Define $\eta$ on $T$ by

$$
\begin{aligned}
\eta(x, y)= & v_{0}\left(\frac{x}{l_{1}}-1\right)\left(\frac{y}{l_{2}}-1\right)-v_{1} \frac{x}{l_{1}}\left(\frac{y}{l_{2}}-1\right) \\
& +v_{2} \frac{x}{l_{1}} \frac{y}{l_{2}}-v_{3}\left(\frac{x}{l_{1}}-1\right) \frac{y}{l_{2}} .
\end{aligned}
$$

It is well known, see, e.g. Ciarlet and Raviart [8] (or by the considerations given below) that with $C$ a universal constant,

$$
\|v-\eta\|_{0, T} \leqslant C L^{2}|v|_{2, T}
$$

Taking $v$ to be of the special form $\omega \chi$ with $\chi$ a bilinear function we obtain

$$
\|\omega \chi-\eta\|_{0, T} \leqslant C L^{2}\|\omega\|_{W_{\infty}^{2}(T)}\|\chi\|_{1, T}+C L^{2}\|\omega\|_{L_{\infty}(T)}\left\|\frac{\partial \chi}{\partial x \partial y}\right\|_{0, T}
$$

Since

$$
\left\|\frac{\partial \chi}{\partial x \partial y}\right\|_{0, T} \leqslant C L^{-1}\|x\|_{1, T}
$$

we have

$$
\|\omega \chi-\eta\|_{0, T} \leqslant C L\|\omega\|_{W_{\infty}^{2}(T)}\|\chi\|_{1, T} .
$$


Consider next $\partial(v-\eta) / \partial x$. We find that

$$
\frac{\partial \eta}{\partial x}=\frac{y}{l_{1} l_{2}}\left(\int_{0}^{l_{1}} \frac{\partial v}{\partial x}\left(s, l_{2}\right) d s\right)-\frac{1}{l_{1}}\left(\frac{y}{l_{2}}-1\right)\left(\int_{0}^{l_{1}} \frac{\partial v}{\partial x}(s, 0) d s\right) .
$$

Thus, $\partial \eta / \partial x$ is constant in $x$ and given as $1 / l_{1}$ times the one dimensional linear interpolant in $y$ of the function

$$
f(y)=\int_{0}^{l_{1}} \frac{\partial v}{\partial x}(s, y) d s
$$

We write

$$
\begin{aligned}
& \frac{\partial \eta}{\partial x}(x, y)-\frac{\partial v}{\partial x}(x, y) \\
& \quad=\left\{\frac{\partial \eta}{\partial x}(x, y)-\frac{1}{l_{1}} f(y)\right\}+\left\{\frac{1}{l_{1}} \int_{0}^{l_{1}} \frac{\partial v}{\partial x}(s, y)-\frac{\partial v}{\partial x}(x, y)\right\} \\
& \quad=I_{1}(x, y)+I_{2}(x, y) .
\end{aligned}
$$

For $I_{1}$, fix $x$, square and integrate with respect to $y$. From well-known properties of linear interpolation we obtain

$$
\int_{0}^{l_{1}}\left|I_{1}(x, y)\right|^{2} d y \leqslant \frac{c}{l_{1}^{2}} l_{2}^{2 j} \int_{0}^{l_{2}}\left|\left(\frac{\partial}{\partial y}\right)^{j} \int_{0}^{l_{1}} \frac{\partial v}{\partial x}(s, y) d s\right|^{2} d y, \quad j=1,2 .
$$

Integrating then with respect to $x$ we arrive at

$$
\left\|I_{1}\right\|_{0, T} \leqslant C l_{2}^{j}\left\|\frac{\partial}{\partial x}\left(\frac{\partial}{\partial y}\right)^{j} v\right\|_{0, T}, \quad j=1,2 .
$$

Consider next $I_{2}(x, y)$. For $y$ fixed, $I_{2}(\cdot, y)$ is the error in a mean value approximation for $\partial v(\cdot, y) / \partial x$. Thus,

$$
\int_{0}^{l_{1}}\left|I_{2}(x, y)\right|^{2} d x \leqslant C l_{1}^{2} \int_{0}^{l_{1}}\left|\frac{\partial^{2} v}{\partial x^{2}}(x, y)\right|^{2} d x
$$

so that

$$
\left\|I_{2}\right\|_{0, T} \leqslant C l_{1}\left\|\frac{\partial^{2} v}{\partial x^{2}}\right\|_{0, T}
$$

From (A.1.3), (A.1.4) and (A.1.5) we conclude that

$$
\left\|\frac{\partial}{\partial \chi}(\eta-v)\right\|_{0, T} \leqslant C\left(l_{2}^{j}\left\|\frac{\partial}{\partial x}\left(\frac{\partial}{\partial y}\right)^{j} v\right\|_{0, T}+l_{1}\left\|\frac{\partial^{2} v}{\partial x^{2}}\right\|_{0, T}\right), \quad j=1,2 .
$$

Again taking $v$ to be $\omega \chi$ with $\chi$ bilinear we have, with $j=2$, that

$$
\left\|\frac{\partial}{\partial x}(\omega \chi-\eta)\right\|_{0, T} \leqslant C\|\omega\|_{w_{\infty}^{3}(T)}\left(l_{2}^{2}\left\|\frac{\partial^{2}}{\partial x \partial y} \chi\right\|_{0, T}+L\|\chi\|_{1, T}\right) .
$$

By use of (A.1.1) we deduce that

$$
\left\|\frac{\partial}{\partial x}(\omega \chi-\eta)\right\|_{0, T} \leqslant C L\|\omega\|_{w_{\infty}^{3}(T)}\|\chi\|_{1, T}
$$


Combining this with its counterpart for $\partial(\omega \chi-\eta) / \partial y$ and with (A.1.2), we have

$$
\|\omega \chi-\eta\|_{1, T} \leqslant C L\|\omega\|_{W_{\infty}^{3}(T)}\|\chi\|_{1, T}
$$

Thus, we have verified A.2 and its counterpart in A.4.

Let us remark that by taking $j=1$ in (A.1.6) we see that the interpolant would work in Lemma 2.1 .

We now consider the inverse assumption A.3(ii); the proof of A.3(i) involves similar ideas and is simpler. Let $\boldsymbol{T}$ be a general rectangle as before. The map

$$
\tilde{x}=\frac{1}{l_{1}} x, \quad \tilde{y}=\frac{1}{l_{2}} y
$$

maps $T$ to the unit square $\widetilde{T}$. Letting $\widetilde{\chi}$ be the transformed function we have

$$
\|\chi\|_{1, T}^{2}=l_{1} l_{2}\left(\|\widetilde{\chi}\|_{0, \tilde{T}}^{2}+\frac{1}{l_{1}^{2}}\left\|\frac{\partial}{\partial \widetilde{x}} \tilde{\chi}\right\|_{0, \tilde{T}}^{2}+\frac{1}{l_{2}^{2}}\left\|\frac{\partial}{\partial \widetilde{y}} \tilde{\chi}\right\|_{0, \widetilde{T}}^{2}\right) .
$$

To be specific, we shall treat the term

$$
I(\tilde{\chi})=\frac{l_{1} l_{2}}{l_{1}^{2}}\left\|\frac{\partial}{\partial \tilde{x}} \tilde{\chi}\right\|_{0, T}^{2} .
$$

By the equivalence of norms on finite dimensional spaces,

$$
I(\tilde{\chi}) \leqslant C \frac{l_{1} l_{2}}{l_{1}^{2}}\|\tilde{\chi}\|_{-p, \tilde{T}}^{2}
$$

Now,

$$
\begin{aligned}
& \|\tilde{\chi}\|_{-p, \tilde{T}}^{2}=\sup _{\tilde{\varphi} \in C^{\infty}(\widetilde{T})} \frac{(\tilde{\chi}, \tilde{\varphi})^{2}}{\sum_{j=0}^{p}\left(\sum_{|\alpha|=j}\left\|\tilde{D}^{\alpha} \tilde{\varphi}\right\|_{0, \tilde{T}}^{2}\right)} \\
& =\sup _{\substack{\sharp C^{\infty}(T) \\
\left(l_{1} l_{2}\right)^{-1}\left(\sum_{j=0}^{p}\left(\sum_{|\alpha|=j}\left(l_{1}^{\alpha} l_{2}^{\alpha} l^{2}\right)^{2}\left\|D^{\alpha} \varphi\right\|_{0, T}^{2}\right)\right)}} \\
& \leqslant\left(l_{1} l_{2}\right)^{-1} \sup _{\substack{\sharp C^{\infty} \\
(T)}} \frac{(\chi, \varphi)^{2}}{\sum_{j=0}^{p} l^{2 j}|\varphi|_{j, T}^{2}}
\end{aligned}
$$

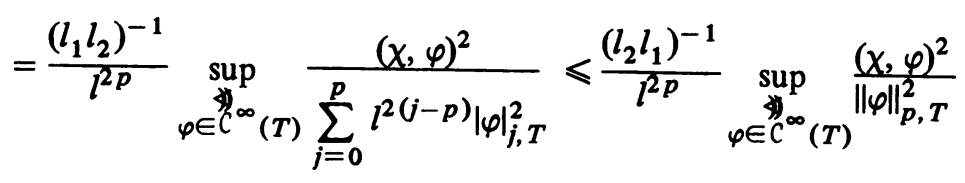

$$
\begin{aligned}
& =\frac{\left(l_{1} l_{2}\right)^{-1}}{l^{2 p}}\|x\|_{-p, T}^{2}
\end{aligned}
$$

By (A.1.8) it follows that

$$
I(\tilde{\chi}) \leqslant C l^{-2(p+1)} \mid\|\chi\|_{-p, T}^{2}
$$


and combining this with similar estimates for the other terms in (A.1.7),

$$
\|\chi\|_{1, T} \leqslant C l^{-(p+1)}\|\| \chi \|_{-p, T}
$$

Thus we have derived the estimate (9.3). The conclusion of the argument is given at the end of Example 2.

Appendix 2. Proof of Lemma 2.2. Assume that for any $v \in \stackrel{\circ}{H}^{1}(\Omega) \cap H^{r}(\Omega)$, there exists a function $\chi \in S^{h}(\Omega)$ such that

$$
\|v-\chi\|_{1, \Omega} \leqslant C h^{r-1}\|v\|_{r, \Omega} .
$$

By Lemma 2.1 (and a simple density argument) this is a consequence of A.1.

To prove Lemma 2.2, we need to construct, for any $v \in \stackrel{\circ}{H}^{1}(\Omega) \cap H^{s}(\Omega), 1 \leqslant$ $s \leqslant r$, a function $\chi \in S^{h}(\Omega)$ such that

$$
\|v-\chi\|_{1, \Omega} \leqslant C h^{s-1}\|v\|_{s, \Omega}
$$

Here the constant $C$ should depend only on $s$.

We shall prove the following: There exists a constant $C$ such that given $v \in$ $\stackrel{\circ}{H}^{1}(\Omega) \cap H^{s}(\Omega)$ and $0<h<1$, there exists $v_{1} \in \stackrel{\circ}{H}^{1}(\Omega) \cap H^{r}(\Omega)$ satisfying

$$
\left\|v-v_{1}\right\|_{1, \Omega} \leqslant C h^{s-1}\|v\|_{s, \Omega}
$$

and

$$
\left\|v_{1}\right\|_{r, \Omega} \leqslant C h^{s-r}\|v\|_{s, \Omega}
$$

Then (A.2.2) would follow upon writing

$$
v-\chi=\left(v-v_{1}\right)+\left(v_{1}-\chi\right)
$$

with $\left\|v_{1}-\chi\right\|_{1, \Omega} \leqslant C h^{r-1}\left\|v_{1}\right\|_{r, \Omega}$, cf. (A.2.1). We shall now give the construction of the function $v_{1}$.

First let $v$ be extended to a function $\tilde{v}$ on $R^{2}$, where the extension is done continuously in $H^{s}$ norms, see Stein [34]. By use of a suitable smoothing operator $S=$ $S(h)$ we have

$$
\|S \tilde{v}-\tilde{v}\|_{1, R^{2}} \leqslant C h^{s-1}\|\widetilde{v}\|_{s, R^{2}}, \quad 1 \leqslant s \leqslant r
$$

and

$$
\left\|S \widetilde{S v \|_{r, R^{2}}} \leqslant C h^{s-r}\right\| \widetilde{v} \|_{s, R^{2}}, \quad 1 \leqslant s \leqslant r .
$$

In terms of the Fourier transform $F$ one may take

$$
F(S \tilde{v})(\xi)= \begin{cases}F(\tilde{v})(\xi), & |\xi| \leqslant 1 / h, \\ 0, & |\xi|>1 / h .\end{cases}
$$

Since $\|\tilde{v}\|_{s, R^{2}}=\left(\int\left(1+|\xi|^{2}\right)^{s}|F \tilde{v}(\xi)|^{2} d \xi\right)^{1 / 2}$ one easily obtains (A.2.5) and (A.2.6).

$$
\text { Let now }
$$

$$
w=\left.S \tilde{v}\right|_{\partial \Omega}
$$


Introduce the following norm for functions defined on $\partial \Omega, k>1 / 2$,

$$
|w|_{k-1 / 2, \partial \Omega}=\inf _{f \in H^{k}(\Omega) ; f=w \text { on } \partial \Omega}\|f\|_{k, \Omega} .
$$

Assume for the moment that for any $w$ as in (A.2.7) we can find an $f_{0} \in H^{r}(\Omega)$ with $f_{0}=w$ on $\partial \Omega$ such that with $C$ a constant independent of $w$,

$$
\left\|f_{0}\right\|_{k, \Omega} \leqslant C|w|_{k-1 / 2, \partial \Omega} \text { for } k=1 \text { and } r \text {. }
$$

We now put $v_{1}=S \tilde{v}-f_{0}$. Note that then $v_{1}$ vanishes on $\partial \Omega$. Let us show how (A.2.3) and (A.2.4) obtain. We have

$$
\left\|v-v_{1}\right\|_{1, \Omega} \leqslant\|\tilde{v}-S \tilde{v}\|_{1, \Omega}+\left\|f_{0}\right\|_{1, \Omega} .
$$

Now by (A.2.9), (A.2.8) and since $\tilde{v}=0$ on $\partial \Omega$,

$$
\left\|f_{0}\right\|_{1, \Omega} \leqslant C|w|_{1 / 2, \partial \Omega}=C|S \tilde{v}-\tilde{v}|_{1 / 2, \partial \Omega} \leqslant C\|S \tilde{v}-\tilde{v}\|_{1, \Omega} .
$$

From this and (A.2.10), using (A.2.5),

$$
\left\|v-v_{1}\right\|_{1, \Omega} \leqslant C\|S \tilde{v}-\tilde{v}\|_{1, \Omega} \leqslant C h^{s-1}\|\widetilde{v}\|_{s, R^{2}} \leqslant C h^{s-1}\|v\|_{s, \Omega} .
$$

This proves (A.2.3).

Next, to prove (A.2.4),

$$
\left\|v_{1}\right\|_{r, \Omega} \leqslant\|S \tilde{v}\|_{r, \Omega}+\left\|f_{0}\right\|_{r, \Omega}
$$

Now by (A.2.9) and (A.2.8),

$$
\left\|f_{0}\right\|_{r, \Omega} \leqslant C|w|_{r-1 / 2, \partial \Omega}=C|S \tilde{v}|_{r-1 / 2, \partial \Omega} \leqslant C\|S \tilde{v}\|_{r, \Omega} .
$$

Hence, by (A.2.6) and (A.2.11),

$$
\left\|v_{1}\right\|_{r, \Omega} \leqslant C\|S \tilde{v}\|_{r, \Omega} \leqslant C h^{s-r}\|\widetilde{v}\|_{s, R^{2}} \leqslant C h^{s-r}\|v\|_{s, \Omega} .
$$

Thus (A.2.4) obtains.

It remains to show that the extension of functions defined on $\partial \Omega$ can be done so as to satisfy (A.2.9). The extension can be done locally, and we shall consider a neighborhood of a corner. Assume first that the corner is convex. By use of an affine mapping we may assume that the interior angle is less than $\pi /(r-1)$. A suitably localized harmonic function $f_{0}$ with boundary values equal to $w$ will then satisfy (A.2.9), see Kondrat'ev [19, Theorem 5.1]. (The result is written down in [19] for $k \geqslant 2$, but is very simple to deduce also for $k=1$.) The affine mapping does not disturb the relation (A.2.9)

For a concave angle, we consider first the exterior wedge, which is then convex, and extend $w$ as above. Thereafter extend the function so obtained to $R^{2}$ to complete the proof. 
1. I. BABUSKA, "Finite element method for domains with corners," Computing, v. 6, 1970, pp. 264-273.

2. I. BABUSKA \& A. K. AZIZ, "Survey lectures on the mathematical foundations of the finite element method," The Mathematical Foundations of the Finite Element Method (A. K. Aziz, Editor), Academic Press, New York, 1972.

3. I. BABUSKKA \& A. K. AZIZ, "On the angle condition in the finite element method," SIAM J. Numer. Anal., v. 13, 1976, pp. 214-226.

4. I. BABUSKKA \& W. RHEINBOLDT, "Mathematical problems of computational decisions in the finite element method," Technical Note TR-426, University of Maryland, 1975.

5. I. BABUSKA \& M. ROSENZWEIG, "A finite element scheme for domains with corners," Numer. Math., v. 20, 1973, pp. 1-21.

6. J. BRAMBLE \& M. ZLAMAL, "Triangular elements in the finite element method," Math. Comp., v. 24, 1970, pp. 809-820.

7. P. G. CIARLET, Numerical Analysis of the Finite Element Method, Séminaire de Mathématiques Supérieures, Presse de l'Université de Montréal, 1976.

8. P. G. CIARLET \& P.-A. RAVIART, "General Lagrange and Hermite interpolation in $R^{n}$ with applications to finite element methods," Arch. Rational Mech. Anal., v. 46, 1972, pp. 177199.

9. Ph. CLEMENT, "Approximation by finite element functions using local regularization," Rev. Française Automat. Informat. Recherche Opérationelle Sér. Rouge, v. 9, 1975, pp. 77-84.

10. J. DOUGLAS, JR., T. DUPONT \& M. F. WHEELER, "An $L^{\infty}$ estimate and a superconvergence result for a Galerkin method based on tensor products of piecewise polynomials," Rev. Française Automat. Informat. Recherche Opérationelle Sér. Rouge, v. 8, 1974, pp. 61-66.

11. S. C. EISENSTAT \& M. H. SCHULTZ, "Computational aspects of the finite element method," The Mathematical Foundations of the Finite Element Method (A. K. Aziz, Editor), Academic Press, New York, 1972, pp. 505-524.

12. J. FREHSE \& R. RANNACHER, "Eine $L^{1}$-Fehlerabschätzung diskreter Grundlösungen in der Methode der finiten Elemente, Tagungsband "Finite Elemente"," Bonn. Math. Schr., 1976.

13. R. GALLAGHER, "Survey and evaluation of the finite element method in fracture mechanics analysis," Proc. First Internat. Conf. on Structural Mech. in Reactor Technology, Berlin, vol. 6, part L, pp. 637-653.

14. P. GRISVARD, "Behavior of the solutions of an elliptic boundary value problem in a polygonal or polyhedral domain," Numerical Solution of Partial Differential Equations-III (B. Hubbard, Editor), Academic Press, New York, 1976, pp. 207-274.

15. S. HILBERT, "A mollifier useful for approximations in Sobolev spaces and some applications to approximating solutions of differential equations," Math. Comp., v. 27, 1973, pp. $81-89$.

16. P. JAMET, "Estimations d'erreur pour des éléments finis droits presque dégénérés," Rev. Française Automat. Informat. Recherche Opérationelle Sér. Rouge, v. 10, 1976, pp. 43-61.

17. R. B. KELLOGG, "Higher order singularities for interface problems," The Mathematical Foundations of the Finite Element Method (A. K. Aziz, Editor), Academic Press, New York, 1972, pp. 589-602.

18. R. B. KELLOGG, "Interpolation between subspaces of a Hilbert space," Technical Note BN-719, University of Maryland, 1971.

19. V. A. KONDRAT'EV, "Boundary problems for elliptic equations in domains with conical or angular points," Trans. Moscow Math. Soc., v. 16, 1967, pp. 227-313.

20. J. L. LIONS \& E. MAGENES, Problèmes aux Limites Non Homogènes et Applications, I, Dunod, Paris, 1968.

21. F. NATTERER, “Über die Punktweise Konvergenz Finiter Elemente," Numer. Math., v. 25, 1975, pp. 67-77.

22. J. NEČAS, Les Méthodes Directes en Théorie des Équations Elliptiques, Masson, Paris, 1967.

23. J. NEČAS, “Sur la coercivité des formes sesquilinéaires elliptiques," Rev. Roumaine Math. Pures Appl., v. 9, 1964, pp. 47-69.

24. J. NITSCHE, " $L_{\infty}$-convergence of finite element approximations," Mathematical Aspects of Finite Element Methods, Rome, 1975.

25. J. NITSCHE, " $L_{\infty}$-convergence for finite element approximation," 2 . Conference on Finite Elements, Rennes, France, May 1975.

26. J. NITSCHE, “On Dirichlet problems using subspaces with nearly zero boundary conditions," The Mathematical Foundations of the Finite Element Method (A. K. Aziz, Editor), Academic Press, New York, 1972, pp. 603-627. 
27. J. NITSCHE \& A. H. SCHATZ, "Interior estimates for Ritz-Galerkin methods," Math. Comp., v. 28, 1974, pp. 937-958.

28. J. NITSCHE \& A. H. SCHATZ, "On local approximation properties of $L_{2}$ projections on spline subspaces," Applicable Anal., v. 2, 1972, pp. 161-168.

29. L. A. OGANESYAN \& L. A. RUKHOVETS, "Variational-difference schemes for linear second-order elliptic equations in a two-dimensional region with piecewise smooth boundary," $\check{Z}$. Vycisl. Mat. i Mat. Fiz., v. 8, 1968, pp. 97-114 = USSR Comput. Math. and Math. Phys., v. 8, 1968, pp. 129-152.

30. T. H. H. PIAN, "Crack elements," Proc. World Congress on Finite Element Methods in Structural Mechanics, Vol. I (J. Robinson, Editor), Robinson \& Assoc., Verwood, Dorset, England, 1975, F.1-F.39.

31. A. H. SCHATZ \& L. B. WAHLBIN, "Interior maximum norm estimates for finite element methods," Math. Comp., v. 31, 1977, pp. 414-442.

32. R. SCOTT, "Optimal $L^{\infty}$ estimates for the finite element method on irregular meshes," Math. Comp., v. 30, 1976, pp. 681-697.

33. L. N. SLOBODECKIII, "Generalized Sobolev spaces and their application to boundary problems for partial differential equations," English transl., Amer. Math. Soc. Transl. (2), v. 57, 1966, pp. 207-276.

34. E. M. STEIN, Singular Integrals and Differentiability Properties of Functions, Princeton Univ. Press, Princeton, N. J., 1970.

35. G. STRANG, "Approximation in the finite element method," Numer. Math., v. 19, 1972, pp. $81-98$.

36. R. W. THATCHER, "The use of infinite grid refinements at singularities in the solution of Laplace's equation," Numer. Math., v. 25, 1976, pp. 163-178.

37. S. V. USPENSKIĬ, "An embedding theorem for S. L. Sobolev's classes of fractional order $W_{p}^{r}$," Dokl. Akad. Nauk SSSR, v. 130, 1960, pp. 992-993 = Soviet Math. Dokl., v. 1, 1960, pp. $132-133$. 